Review

\title{
Novel Approaches for the Treatment of Necrotic Immature Teeth Using Regenerative Endodontic Procedures: A Systematic Review and Meta-Analysis
}

\author{
María Eugenia Marcela Castro-Gutiérrez ${ }^{1}$, Liliana Argueta-Figueroa ${ }^{2, * \mathbb{D}}$, Gisela Fuentes-Mascorro ${ }^{3}$, \\ Adriana Moreno-Rodríguez ${ }^{4}$ and Rafael Torres-Rosas ${ }^{5, *(D)}$ \\ 1 Facultad de Medicina, Universidad Autónoma "Benito Juárez" de Oaxaca, ExHacienda de Aguilera S/N, \\ Calzada, San Felipe del Agua, Oaxaca 68120, Mexico; marcecastrog@hotmail.com \\ 2 Cátedras CONACyT-Facultad de Odontología, Universidad Autónoma “Benito Juárez" de Oaxaca, \\ Avenida Universidad S/N, Ex-Hacienda 5 Señores, Oaxaca 68120, Mexico \\ 3 Laboratorio de Investigación en Reproducción Animal (LIRA), Universidad Autónoma Benito Juárez, \\ Avenida Universidad S/N, Ex-Hacienda de Cinco Señores, Oaxaca 68120, Mexico; lirauabjo@gmail.com \\ 4 Laboratorio 16, Facultad de Ciencias Químicas, Universidad Autónoma "Benito Juárez" de Oaxaca, \\ Avenida Universidad S/N, ExHacienda de Cinco Señores, Oaxaca 68120, Mexico; arimor10@gmail.com \\ 5 Laboratorio de Inmunología Asociado al Centro de Estudios en Ciencias de la Salud y la Enfermedad, \\ Facultad de Odontología, Universidad Autónoma "Benito Juárez" de Oaxaca, Avenida Universidad S/N, \\ ExHacienda 5 Señores, Oaxaca 68120, Mexico \\ check for \\ * Correspondence: liliana.argueta@conacyt.mx (L.A.-F.); rtorres.cat@uabjo.mx (R.T.-R.)
}

updates

Citation: Castro-Gutiérrez, M.E.M.; Argueta-Figueroa, L.;

Fuentes-Mascorro, G.;

Moreno-Rodríguez, A.; Torres-Rosas,

R. Novel Approaches for the

Treatment of Necrotic Immature

Teeth Using Regenerative Endodontic Procedures: A Systematic Review and Meta-Analysis. Appl. Sci. 2021, 11, 5199. https://doi.org/10.3390/

11115199

Academic Editor: Ivana Miletić

Received: 8 May 2021

Accepted: 31 May 2021

Published: 3 June 2021

Publisher's Note: MDPI stays neutral with regard to jurisdictional claims in published maps and institutional affiliations.

Copyright: (c) 2021 by the authors. Licensee MDPI, Basel, Switzerland. This article is an open access article distributed under the terms and conditions of the Creative Commons Attribution (CC BY) license (https:// creativecommons.org/licenses/by/ $4.0 /)$.

\begin{abstract}
The induction of a blood clot allowing apical revascularization with a subsequent apical closure was reported several decades ago. Recent research has been focused on examining different scaffolds for regenerative endodontic procedures (REPs). The aim of this study was to analyze the main outcomes in novel REP reported in the literature. The search was conducted using six databases. Only clinical trials aiming at the study of scaffolds for REP in the treatment of necrotic immature teeth were included. The risk of bias was assessed by the Risk of Bias 2 tool. Additionally, the quality of the included studies was assessed using the Grading of Recommendations Assessment, Development, and Evaluation (GRADE) criteria. A summary of individual studies and a meta-analysis were performed. Relative risks of data from clinical success and root length changes were used from the studies, and these were combined using a random-effects meta-analysis. The meta-analysis results reveal that the main outcomes showed no differences between the patients who received REP with scaffolds or only REP. Limitations include that the certainty of evidence was low and that there was significant heterogeneity in the scaffolds used in the included studies. The data suggest that the blood clot remains as the gold standard for REP.
\end{abstract}

Keywords: immature teeth; dental pulp; regeneration

\section{Introduction}

Apexification used to be the conventional treatment used for necrotic immature teeth (NIT). However, the characteristics of these teeth, which are fragile due to the thin thickness of the root canal dentin, together with the anatomy of an open apex, make it difficult to properly instrumentation, and complete canal obturation without exceeding the periapical tissues [1].

Since the 1960s, it has been reported that the induction of apical closure due to the formation of hard tissue was achieved by the closure of the root canal using a plug containing calcium hydroxide $(\mathrm{Ca}(\mathrm{OH}) 2)$. To explain that phenomena, it was hypothesized that the epithelial cell rests of Malassez persist throughout life and are capable of resuming the formation of mineralized tissue on the root surface once the source of infection has been removed [2]. However, there are long-term effects of the aforementioned technique 
using $\mathrm{Ca}(\mathrm{OH})_{2}$ for NIT treatment. Its disadvantages have been widely debated in the literature, as the need for several material replacements, with the consequent possibility of recontamination of the root canal during the treatment period and the weakening of the root dentin [3]. Another concern was that $\mathrm{Ca}(\mathrm{OH})_{2}$ could cause damage to the undifferentiated cells present in the ligament or the remnants of mesenchymal tissue, and preventing the root maturation [4].

On the other hand, recently, the introduction of the regenerative endodontic procedures (REPs) implies the induction of a blood clot (BC) which could allow apical revascularization, and would lead to a subsequent apical closure. However, the idea of placing a plug of some material on a BC implies the reconsideration of certain well-established endodontic principles; likewise, the procedure to generate such as a clot (inserting a file beyond the apical radiographic limits) involves that endodontic paradigm changes [5]. Due to this, REP has emerged as an alternative for the treatment of NIT. The goal of REP for the treatment of NIT is to provide a convenient microenvironment for the reestablishment of a rich blood supply and stem cells that allow the root healing [6]. This is possible to achieve due to the fast that the apical papilla and its rich resident stem cell population are capable of surviving infection and advanced apical periodontitis [7]. In REP, the periapical tissues are intentionally damaged to promote bleeding and a fibrin clot acts as a natural scaffold allowing the proliferation and differentiation of dentin-pulp stem cells [8]. Unlike traditional apexification or the use of apical barriers, the success of REP allows increasing both the length of the root and the thickness of the dentin root walls. The vascularization is crucial for the supply of the cells and the scaffolds inside the root canal and for that reason, it is important to cause enough bleed. An adequate $\mathrm{BC}$ also prevents the sealing material from collapsing. In addition to $\mathrm{BC}$, it has been postulated that the use of the scaffolds is crucial because it provides a structural organization for stem cells to distribute, proliferate, and differentiate into odontoblasts-like cells [9].

Therefore, recent research has focused on examining different scaffolding models for REP. However, given the range of possibilities, it is important to consider the clinical success of the different scaffolds. REP should be carried out based on evidence-based dentistry. Several reviews of the literature have been published to date, however, the included studies come from case series, clinical cases, or observational studies with very small samples (less than ten participants), which directly impacts the reliability of the conclusions. On the other hand, these reviews also did not present the assessment of risk of bias and the quality assessment of the included studies, as well as a quantitative analysis. The importance of synthesizing evidence from clinical trials with the risk of bias and quality assessments allows that the results of the present systematic review and meta-analysis to be extrapolated, that is, to have external validity.

Accordingly, this review aimed to determine the effectiveness (measured as increase of the root length, or changes of the apical diameter, or clinical success) of the different REPs with scaffolds in comparison with the apexification or REP using only BC as control groups, in patients with NIT from clinical trials.

\section{Materials and Methods}

\subsection{Study Design}

The types of studies included in the systematic review were randomized controlled trials and non-randomized clinical trials. On the other hand, we excluded case reports, case series, letters, comments, short communications, pilot studies (ten patients or less), animal studies, in vitro studies, and literature reviews.

\subsection{Eligibility Criteria and Participant Characteristics of Studies}

The eligibility criteria for the included studies were defined considering the PICO (Population, Intervention, Comparison, and Outcome.) definitions as follows:

Population: Patients with NIT. 
Interventions: REPs including: 1. Stem Cells, 2. Platelet-rich plasma, 3. Scaffolds, or 4. Growth factors.

Comparator: Apexification, or REPs using only BC, or some variant of the studied intervention as the control group.

Outcomes: Root length, or apical diameter, or clinical success (resolution of symptomatology). The keywords and algorithms used for the search strategy are shown in Supplementary Materials Table S1.

Question of the review: What is the effectiveness (measured as increase of the root length, or changes of the apical diameter, or clinical success) of the different REP with scaffolds in comparison with REPs using only BC as control groups, in patients with NIT from clinical trials?

\subsection{Database Search}

The search was performed on the electronic databases PubMed, Scholar Google, Clinical Trials, ProQuest, Web of science, and Scopus during the last months of 2020 and was run again in May of 2021. A manual search of the bibliographical references of the included studies in the review was performed. The manual search was achieved through bibliographical references of the studies included. The only restriction in the search was the language of the publication (only full-text articles in English or Spanish).

\subsection{Study Selection}

Studies for their inclusion in the review were chosen by reading the title and abstract of each record identified by the search. The full-text articles of the selected studies that matched eligibility criteria were retrieved for a more detailed review. In the event of reviewing a full-text that did not fully meet the eligibility criteria, those studies were excluded with reasons.

\subsection{Data Collection Process and Data Items}

A standardized Microsoft Excel worksheet was prepared for the registration of the relevant data of all the studies included in the systematic review. Such as participant demographics and baseline characteristics, methodology, time intervals in which the effect of the intervention is measured, symptomatology, root measures at baseline, and followups. Two reviewers were responsible for data extraction (M.E.M.C.-G., and G.F.-M.). The study researchers were contacted for missing data or additional details via email.

\subsection{Risk of Bias in Individual Studies and Quality Assessment}

Two reviewers (L.A.-F., and R.T.-R.) following the recommendations from the Cochrane Handbook for Systematic Reviews of Interventions (Chapter 8) [10] and Risk of Bias 2 (RoB 2) tool evaluated the risk of bias of the included studies [11]. Additionally, two reviewers (M.E.M.C.-G., and A.M.-R.) assessed the quality of the studies (certainty in the evidence) using the Grading of Recommendations Assessment, Development, and Evaluation (GRADE) criteria.

\subsection{Strategy for Data Synthesis}

The qualitative and quantitative synthesis from the data of the included articles were performed. The heterogeneity between the measured effects from the studies was evaluated. The data were grouped according to clinical success and increase of root length outcomes. For the meta-analysis, the data collected from the selected studies were carefully analyzed to determine whether the studies can be grouped. Relative risks were used from the individual studies and these were combined using a random-effects metaanalysis. Moreover, 95\% confidence intervals (CI) and two-sided values were calculated. The heterogeneity between the measures of effect from the studies was evaluated using the $\mathrm{I}^{2}$ statistic and was considered an $\mathrm{I}^{2}$ value greater than $50 \%$ as indicative of substantial heterogeneity. 


\section{Results}

A total of 4603 records were identified in the search. After the removal of duplicate records, the selection was based on the title and abstract of these records. Eighteen full-text articles were analyzed, of these, eight studies [12-19] were excluded with reasons; therefore, ten articles were included in this review as shown in Figure 1. The overall risk of bias was moderate due to bias in the selection of the reported results $(100 \%)$, deviations from intended interventions (70\%), and randomization process $(30 \%)$ domains as is shown in Figure 2.

The main REPs identified in the articles were the use of platelet-rich plasma (PRP), followed by platelet-rich plasma (PRF) (six and five articles, respectively). Minority REPs were platelet-pellet (PP), fibroblast growth factor (FGF), and Bio-guide collagen membrane (CMB) (only one article of each one).
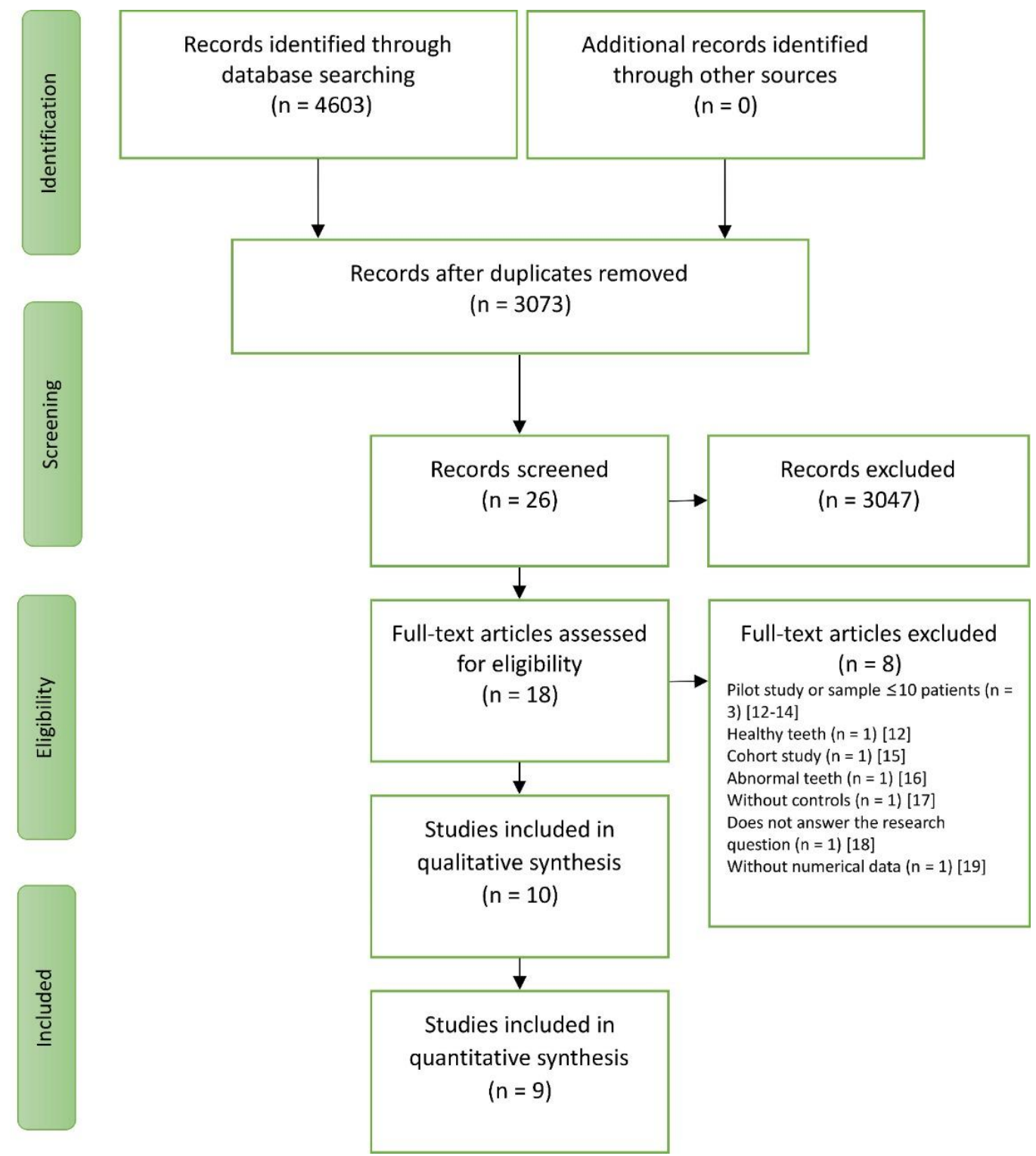

Figure 1. PRISMA Flow Diagram for systematic review. 


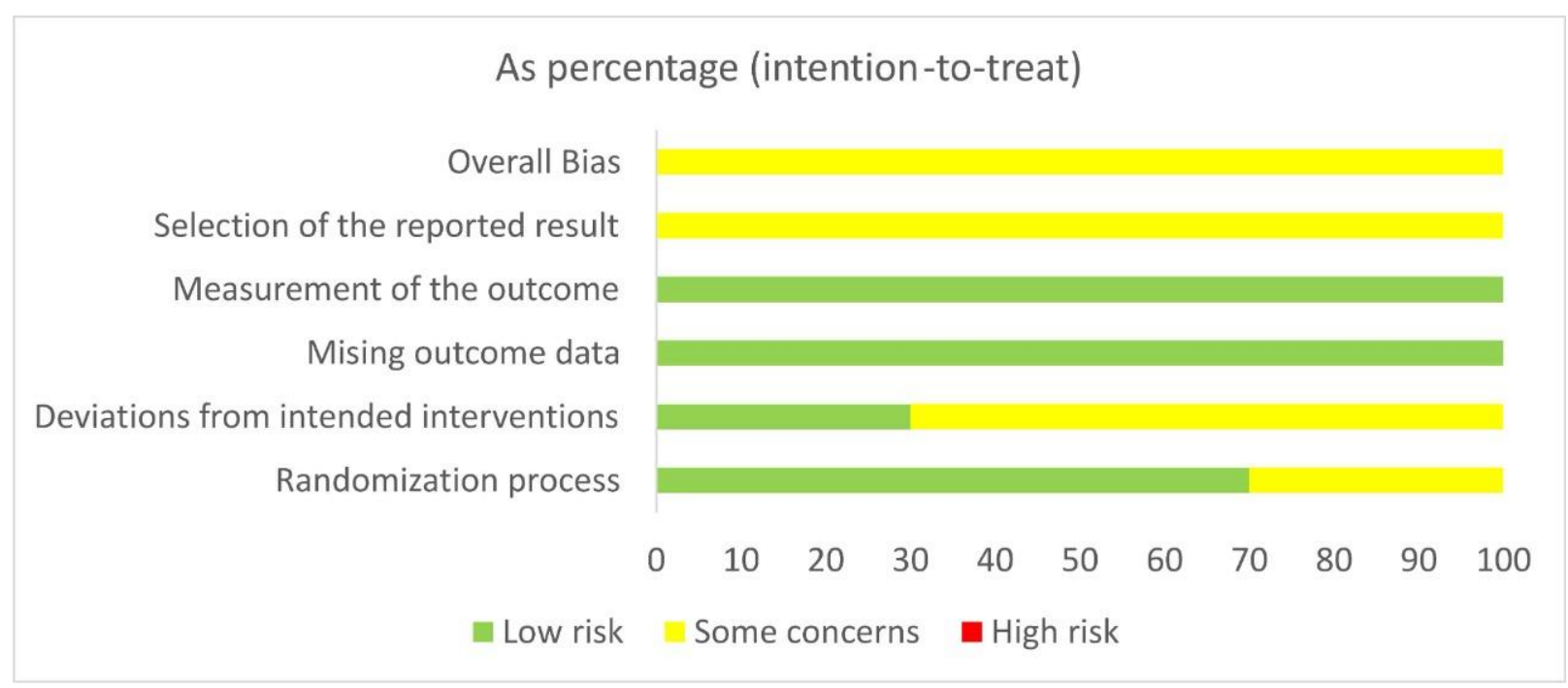

(a)

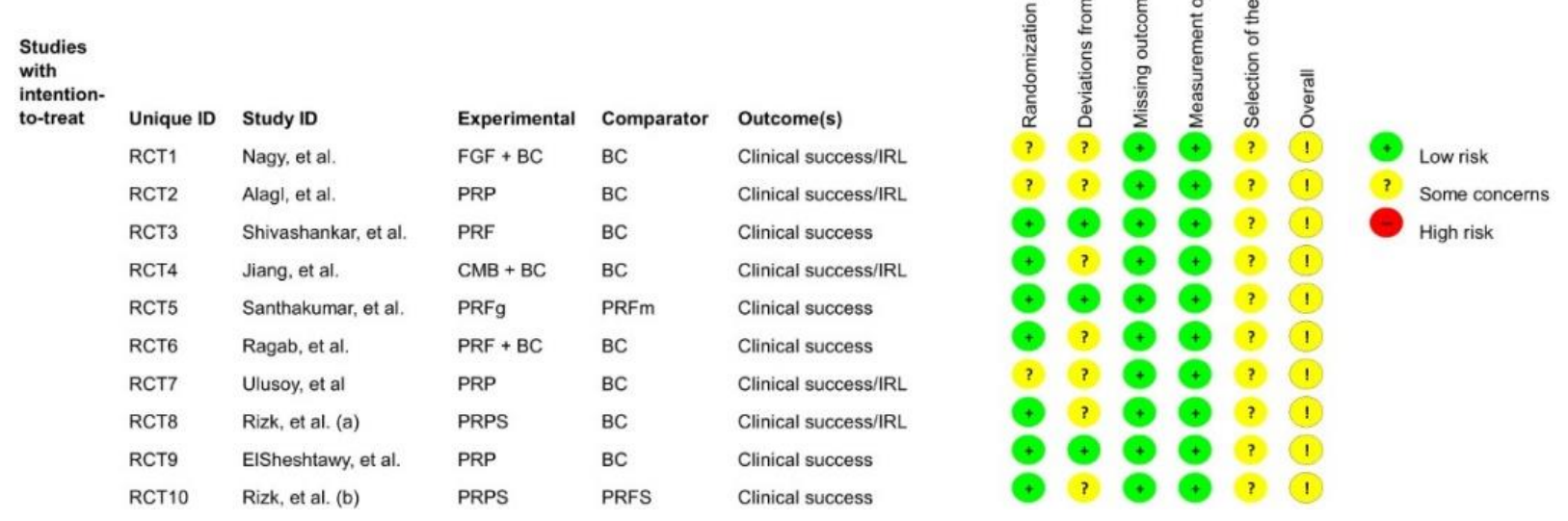

(b)

Figure 2. (a) Percentage of risk of bias by domain; (b) Risk of bias for individual studies included in the systematic review: Increase root length (IRL), basic fibroblast growth factor (FGF), regenerative endodontic protocol of blood clot (BC), collagen membrane bio-guide (CMB), platelet-rich plasma (PRP), platelet-rich fibrin gel (PRFg), platelet-rich fibrin membrane (PRFm), platelet-rich plasma scaffold (PRPS), platelet-rich fibrin scaffold (PRFS).

The studies were grouped according to the outcome and analyzed under the following subheadings: clinical success, root length, changes in apical diameter, dentin thickness, bone density, and pulp sensibility. The characteristics and the results of the individual studies are summarized in Table 1.

\subsection{Clinical Success}

In most studies assessed, the clinical success was defined with the following criteria: lack of symptoms such as pain, swelling, tenderness to percussion, and mobility. In one study, the success rates were scored as 0 (failure), 1 (resolution of the disease), or 2 (resolution of the disease + radiographic root development and positive response to cold testing and electric pulp testing). 
Table 1. Characteristics of the individual studies and their results.

\begin{tabular}{|c|c|c|c|c|c|c|c|}
\hline Article & Patients Age/Sex & Intervention & Medication & $\begin{array}{l}\text { Preparation } \\
\text { of Scaffold }\end{array}$ & Restoration & Outcomes & Results \\
\hline \multirow{16}{*}{ Nagy et al. [20] } & \multirow{16}{*}{$\begin{array}{c}\text { Immature } \\
\text { necrotic permanent } \\
\text { maxillary central } \\
\text { incisors. }(n=36) \\
\text { Age: } \\
\text { 9-13 years } \\
\text { G1: } \mathrm{M}(\mathrm{n}=7), \mathrm{F}(\mathrm{n}=5) \\
\text { G2: } \mathrm{M}(\mathrm{n}=5), \mathrm{F}:(\mathrm{n}=7) \\
\text { G3: } \mathrm{M}(\mathrm{n}=6), \mathrm{F}(\mathrm{n}=5)\end{array}$} & \multirow{16}{*}{$\begin{array}{c}\text { G1: MTA } \\
(\mathrm{n}=12) \\
\text { G2: } \mathrm{BC} \\
(\mathrm{n}=12) \\
\text { G3: } \mathrm{BC}+\mathrm{FGF}(\mathrm{n}=12)\end{array}$} & \multirow{16}{*}{$\begin{array}{l}\text { Irrig: } 2.6 \% \mathrm{NaClO} \\
\text { ABX (MTZ, } \\
\text { CPO and } \\
\text { doxycycline) } \\
\text { TS: Coltosol F } \\
\text { Time: } 3 \text { weeks }\end{array}$} & \multirow{16}{*}{$\begin{array}{l}150 \mathrm{mg} \text { FGF }+300 \mathrm{~mL} \\
\text { phosphate-buffered saline } \\
\text { + 2-mg dried gelatin } \\
\text { hydrogel sheet. }\end{array}$} & \multirow{16}{*}{$\begin{array}{l}\text { MTA and } \\
\text { Adhesive } \\
\text { composite resin. }+\end{array}$} & \multirow{16}{*}{$\begin{array}{c}\text { Clinical } \\
\text { success (CLS) } \\
\text { Increase in Root Length (IRL) } \\
\text { Decrease in the Apical } \\
\text { Diameter (DAD) } \\
\text { Increase in Root Thickness (IRT) } \\
\text { Decrease in Bone Density (DBD) }\end{array}$} & CLS: \\
\hline & & & & & & & $\begin{array}{l}\text { G1: } 100 \% 12 / 12 \\
\text { G2: } 90 \% 11 / 12 \\
\text { G3: } 80 \% 10 / 12\end{array}$ \\
\hline & & & & & & & IRL: n (\%) \\
\hline & & & & & & & 3 months: \\
\hline & & & & & & & $\begin{array}{c}\text { G1: } 0 \pm 0(0 \pm 0)^{\mathrm{A}}, \\
\text { G2: } 0.3 \pm 0.2(3 \pm 3.5)^{\mathrm{B}}, \\
\text { G3: } 0.3 \pm 0.2(3 \pm 2)^{\mathrm{B}}, \\
\left(p<0.001^{*}\right) .\end{array}$ \\
\hline & & & & & & & 6 months: \\
\hline & & & & & & & $\begin{array}{c}\text { G1: } 0 \pm 0(0 \pm 0)^{\mathrm{A}}, \\
\text { G2: } 0.6 \pm 0.3(5.3 \pm 2.6)^{\mathrm{B}}, \\
\text { G3: } 0.6 \pm 0.3(6.1 \pm 3)^{\mathrm{B}}, \\
(p<0.001 *) .\end{array}$ \\
\hline & & & & & & & 12 months: \\
\hline & & & & & & & $\begin{array}{c}\text { G1:0 } \pm 0(0 \pm 0)^{\mathrm{A}}, \\
\text { G2: } 0.8 \pm 0.5(7.6 \pm 4.7)^{\mathrm{B}}, \\
\text { G3: } 1 \pm 0.5(9.9 \pm 4.9)^{\mathrm{B}}, \\
\left(p<0.001^{*}\right) .\end{array}$ \\
\hline & & & & & & & 18 months: \\
\hline & & & & & & & $\begin{array}{c}\text { G1: } 0 \pm 0(0 \pm 0)^{\mathrm{A}}, \mathrm{G} 2: 1.2 \pm 0.5 \\
(11.8 \pm 4.9)^{\mathrm{B}} \\
\text { G3: } 1.3 \pm 0.5(12.4 \pm 4.7)^{\mathrm{B}} \\
(p<0.001 *) .\end{array}$ \\
\hline & & & & & & & DAD: n (\%) \\
\hline & & & & & & & 3 months: \\
\hline & & & & & & & $\begin{array}{c}\text { G1: } 0 \pm 0(0 \pm 0)^{\mathrm{A}}{ }^{\prime} \\
\text { G2: } 0.08 \pm 0.04(6.4 \pm 3.2)^{\mathrm{B}} \\
\text { G3: } 0.06 \pm 0.04(5.7 \pm 3.7)^{\mathrm{B}},(p<< \\
0.001) .\end{array}$ \\
\hline & & & & & & & 6 months: \\
\hline & & & & & & & $\begin{array}{c}\text { G1: } 0 \pm 0(0 \pm 0)^{\mathrm{A}} \\
\text { G2: } 0.22 \pm 0.11(17.6 \pm 8.8)^{\mathrm{B}} \\
\text { G3: } 0.15 \pm 0.08(12.6 \pm 6.7)^{\mathrm{B}^{\prime}} \\
(p<0.001) .\end{array}$ \\
\hline
\end{tabular}


Table 1. Cont.

Article

Patients Age/Sex

Intervention

Medication

Preparation
of Scaffold

Restoration

Outcomes

Results

12 months:

$\mathrm{G} 1 \cdot 0 \pm 0(0+0) \mathrm{A}$

C2. $0.67 \pm 0(0 \pm 0)^{\mathrm{A}}$

G3: $0.59 \pm 0.41(29.9 \pm 20.7)^{B}$ $(p<0.001)$.

18 months:

G1: $0 \pm 0(0 \pm 0)^{\mathrm{A}}$

G2: $0.8 \pm 0.3(50.5 \pm 18.9)^{\mathrm{B}}, \mathrm{G} 3: 0.9$ $\pm 0.2(44.3 \pm 9.8)^{\mathrm{B}}$

$(p<0.0001)$.

\begin{tabular}{c}
\hline IRT: $\mathbf{n}(\%)$ \\
\hline 3 months: \\
\hline
\end{tabular}

G1: $0 \pm 0(0 \pm 0)^{\mathrm{A}}$,

G2: $0.04 \pm 0.03(1.8 \pm 1.3)^{\mathrm{B}^{\prime}}, \mathrm{G} 3: 0.06$ $\pm 0.01(2.3 \pm 1.4)$

\begin{tabular}{c}
$(p<0.001)$. \\
\hline 6 months:
\end{tabular}

G1: $0 \pm 0(0 \pm 0)^{\mathrm{A}}$

G2: $0.14 \pm 0.03(5.8 \pm 1.2)^{\mathrm{B}^{\prime}}, \mathrm{G} 3: 0.11$ $\pm 0.04(4.5 \pm 1.6)^{\mathrm{B}}$

$(p<0.001)$.

$\frac{(p<0.001) .}{12 \text { months: }}$

G1: $0 \pm 0(0 \pm 0)^{\mathrm{A}}$

G2: $0.21 \pm 0.08(8.4 \pm 3.2)^{\mathrm{B}}, \mathrm{G} 3: 0.2$ $(p<0.001)$.

18 months:

G1: $0 \pm 0(0 \pm 0)^{\mathrm{A}}$

G2: $0.32 \pm 0.12(12.7 \pm 4.7)^{\text {В }}, \mathrm{G} 3$ :

$0.29 \pm 0.09(11.6 \pm 3.6)^{B^{\prime}}$

DBD: (\%)

3 months:

G1: $4.47 \pm 5.6$

G3: $2.51 \pm 2.1(p=0.2664)$ 
Table 1. Cont.

\begin{tabular}{|c|c|c|c|c|c|c|c|}
\hline Article & Patients Age/Sex & Intervention & Medication & $\begin{array}{l}\text { Preparation } \\
\text { of Scaffold }\end{array}$ & Restoration & Outcomes & Results \\
\hline & & & & & & & 6 months (\%): \\
\hline & & & & & & & $\begin{array}{c}\text { G1: } 8.71 \pm 3.2, \\
\text { G2: } 6.43 \pm 2.1 \\
\text { G3: } 5.26 \pm 3.7(p=0.1941) .\end{array}$ \\
\hline & & & & & & & 12 months (\%): \\
\hline & & & & & & & $\begin{array}{c}\text { G1: } 11.25 \pm 7.8 \\
\text { G2: } 10.91 \pm 6.2 \\
\text { G3: } 9.56 \pm 5.8,(p=0.8073) .\end{array}$ \\
\hline & & & & & & & $\begin{array}{c}\text { G1:14.61 } \pm 7.1, \\
\text { G2: } 12.77 \pm 5.5 \\
\text { G3: } 12.21 \pm 6,(p=0.6191) .\end{array}$ \\
\hline \multirow{6}{*}{ Alagl et al. [1] } & \multirow{6}{*}{$\begin{array}{l}\text { Young, immature teeth and } \\
\text { single-rooted with apical } \\
\text { periodontitis. } \\
\text { Patients } \\
\text { Age: } 8 \text {-11 years } \\
\text { M }(\mathrm{n}=9) \\
\mathrm{F}(\mathrm{n}=6) \\
\text { Teeth } \\
(\mathrm{n}=30)\end{array}$} & \multirow{6}{*}{$\begin{array}{l}\text { G1: PRP } \\
(\mathrm{n}=15) \\
\text { G2: } \mathrm{BC} \\
(\mathrm{n}=15)\end{array}$} & \multirow{6}{*}{$\begin{array}{c}\text { Irrig: } 1.5 \% \mathrm{NaCl} \text { and } \\
0.12 \% \\
\text { chlorhexidine. } \\
\text { ABX (MTZ, CPO and } \\
\text { MNO) } \\
\text { TS: zinc oxide- } \\
\text { eugenol cement. } \\
\text { Time: } 3 \text { weeks }\end{array}$} & \multirow{6}{*}{$\begin{array}{l}\text { PRP + equal volumes of } \\
\text { sterile saline } \\
\text { solution containing } 10 \% \\
\text { calcium chloride and } \\
\text { sterile bovine thrombin } \\
\text { (100 U/mL). }\end{array}$} & \multirow{6}{*}{$\begin{array}{c}\text { MTA and } \\
\text { Glass ionomer } \\
\text { cement (Fuji VII; GC, } \\
\text { Tokyo, } \\
\text { Japan), and } \\
\text { composite resin (Filtek } \\
\text { Z350; } \\
\text { 3M ESPE, } \\
\text { St. Paul, MN) }\end{array}$} & \multirow{6}{*}{$\begin{array}{c}\text { Clinical } \\
\text { success (CLS) } \\
\text { Vitality } \\
\text { test (VT) } \\
\text { Lesion size } \\
\text { Bone density } \\
\text { Root length } \\
\text { Complete } \\
\text { apical closure } \\
\text { Pre-and } \\
\text { post-treatment comparison of } \\
\text { parameters (CPPP) }\end{array}$} & $\begin{array}{c}\text { CLS: } \\
\text { G1:100\% } \\
\text { G2:100\% }\end{array}$ \\
\hline & & & & & & & $\begin{array}{l}\text { VT: } \\
\text { G1: Positive: } 12 \text { teeth, } \\
\text { Negative: } 3 \text { teeth } \\
\text { G2: Positive: } 6 \text { teeth, } \\
\text { Negative: } 9 \text { teeth }\end{array}$ \\
\hline & & & & & & & $\begin{array}{c}\text { Bone density (HU): } \\
\text { Mean (SD) } \\
\text { G1: } 365.3(127.50) \\
\text { G2: } 316.4(123.31),(p=0.132) .\end{array}$ \\
\hline & & & & & & & $\begin{array}{c}\text { Root length (mm): } \\
\text { Mean (SD) } \\
\text { G1: } 1.06(0.62) \\
\text { G2: } 0.502(0.42) \\
\left(p=0.004^{*}\right)\end{array}$ \\
\hline & & & & & & & $\begin{array}{l}\text { Complete apical closure: } \\
\text { G1: } 14 / 15 \\
\text { G2: } 8 / 15\end{array}$ \\
\hline & & & & & & & $\begin{array}{c}\text { CPPP: } \\
\text { G1: mean (SD) } \\
\text { Lesion size }(\mathrm{mm}) \text { initial:3.92 }(1.46) \text {, } \\
\text { follow up: } 1.50(1.08),\left(p=0.001{ }^{*}\right)\end{array}$ \\
\hline
\end{tabular}


Table 1. Cont.

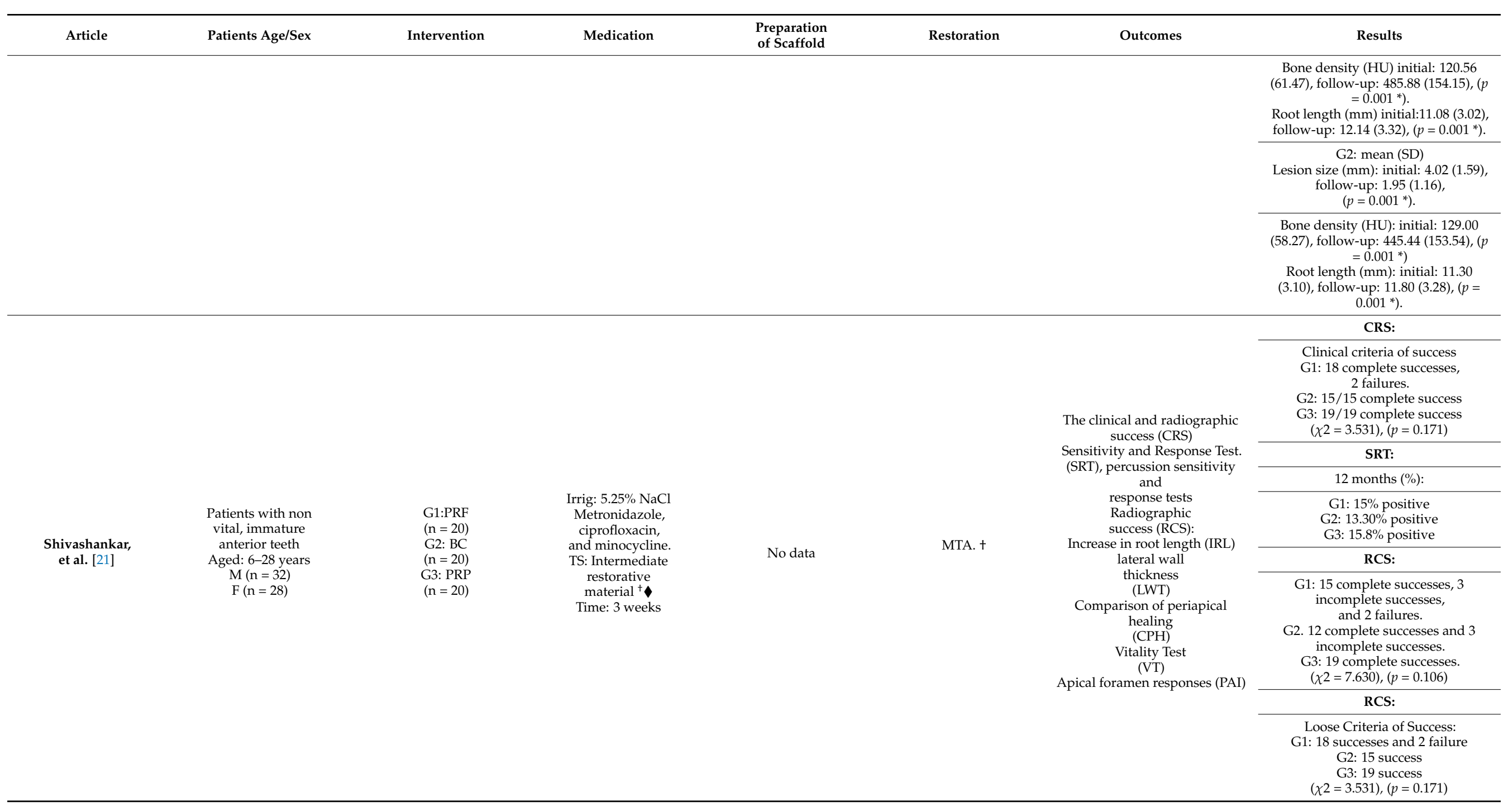


Table 1. Cont.

Article

Patients Age/Sex

Intervention
Preparation
of Scaffold
Restoration

Outcomes

Results

ct Criteria of Success:

G1: 15 successes and 5 failure

G2: 12 successes and 3 failure

G3: 19 success
$(\chi 2=5.268),(p=0072)$

IRL: at 12 months

G1: $30 \%$ good,

$35 \%$ satisfactory,

and $35 \%$ no change

G2: $26.7 \%$ good,

60\% satisfactory

and $13.3 \%$ no change

G3: $26.3 \%$ good,

and $15.8 \%$ no chang

LWT: at 12 months

G1: $30 \%$ good,

$40 \%$ satisfactory

and $30 \%$ no change

G2: $20 \%$ good,

73.3\% satisfactory

G3: $26.3 \%$ good,

G3: $26.3 \%$ good,

and 15.8 no change.

CPH: Mean \pm SD

6th Month

G1: $3.20 \pm 0.410(\mathrm{n}=20)$
G2: $2.93 \pm 0.458(\mathrm{n}=15)$

G3: $2.74 \pm 0.562(\mathrm{n}=19)$

$(F=4.549)(p=0.015)$

12th Month

G1: $1.85 \pm 1.040(\mathrm{n}=20)$

G2: $2.07 \pm 0.594(\mathrm{n}=15)$

$(F=4.524)(p=0.016)$

VT: 12th Month

1: $15 \%$ positive,
$85 \%$ negative

85\% negative

G2: $13.3 \%$ positive
86.7 negative

86.7 negative
G3: $15.8 \%$ positive,

84.2 negative 
Table 1. Cont.

\begin{tabular}{|c|c|c|c|c|c|c|c|}
\hline Article & Patients Age/Sex & Intervention & Medication & $\begin{array}{l}\text { Preparation } \\
\text { of Scaffold }\end{array}$ & Restoration & Outcomes & Results \\
\hline & & & & & & & $\begin{array}{c}\text { PAI: } 12 \text { th Month } \\
\text { G1: Type } 1(\mathrm{n}=2) 10 \% \text {, Type } 2(\mathrm{n}=2) \\
\text { 10\%, Type } 3(\mathrm{n}=10) 50 \% \text {, Type } 4(\mathrm{n}= \\
\text { 1) } 5 \% \text {, Type } 5(\mathrm{n}=1) 5 \% \text {, No Change } \\
\text { (n = 4) } 20 \% \text {, Total }(\mathrm{n}=20) 100 \% \text {. } \\
\text { G2: Type } 1(\mathrm{n}=2) 13.3 \% \text {, Type } 2(\mathrm{n}= \\
\text { 3) } 20 \%, \text { Type } 3(\mathrm{n}=8) 53.3 \% \text {, Type } 4 \\
(\mathrm{n}=0) 0 \% \text {, Type } 5(\mathrm{n}=1) 6.7 \% \text {, No } \\
\text { Change }(\mathrm{n}=1) 6.7 \% \text {, } \\
\text { Total }(\mathrm{n}=15) 100 \% . \\
\text { G3: Type } 1(\mathrm{n}=2) 10.5 \%, \text { Type } 2(\mathrm{n}= \\
\text { 2) } 10.5 \% \text {, Type } 3(\mathrm{n}=12) 63.2 \% \text {, Type } \\
\text { 4(n=0) } 0 \%, \text { Type } 5(\mathrm{n}=0) 0 \% \text {, No } \\
\text { Change }(\mathrm{n}=3) 15.8 \%, \\
\text { Total }(\mathrm{n}=19) 100 \% .\end{array}$ \\
\hline \multirow{8}{*}{ Jiang et al. [22] } & \multirow{8}{*}{$\begin{array}{c}\text { Pediatric Patients with } \\
\text { immature teeth } \\
\text { affected by pulp and } \\
\text { periapical diseases. } \\
(\mathrm{n}=46) \\
\mathrm{G} 1: \\
\text { Age: } 10.3 \pm 1.9 \\
\text { M }(\mathrm{n}=9), \mathrm{F}(\mathrm{n}=11) \\
\mathrm{G} 2: \\
\text { Age: } 9.82 \pm 1.5 \\
\text { M }(\mathrm{n}=8) \\
\mathrm{F}(\mathrm{n}=12)\end{array}$} & \multirow{8}{*}{$\begin{array}{c}\text { G1: } \mathrm{BC}+\mathrm{CMB}(\mathrm{n}=23) \\
\text { G2: } \mathrm{BC} \\
(\mathrm{n}=23)\end{array}$} & \multirow{8}{*}{$\begin{array}{l}\text { Irrig: } 1.25 \% \mathrm{NaCl} \\
\mathrm{Ca}(\mathrm{OH})_{2} \text { paste } \\
\text { TS: } 3-4 \mathrm{~mm} \text { glass } \\
\text { ionomer } \\
\text { Time: } 2 \text { weeks }\end{array}$} & \multirow{8}{*}{$\begin{array}{l}\text { Bio-Gide collagen } \\
\text { membrane. }\end{array}$} & \multirow{8}{*}{$\begin{array}{c}\text { MTA and } \\
\text { Composite resin (3M } \\
\text { ESPE, Irvine, CA; } 3-4 \\
\text { mm) }\end{array}$} & \multirow{8}{*}{$\begin{array}{l}\text { Clinical success (CLS) } \\
\text { Increase in root length (IRL) } \\
\text { Change in } \\
\text { root length } \\
\text { Increase in dentin wall } \\
\text { thickness in the apical third of } \\
\text { the root (IWTA) } \\
\text { Increase in dentin wall } \\
\text { thickness in the middle third of } \\
\text { the root (IWTM) } \\
\text { Narrowing of } \\
\text { apicalforamen width (NAF) } \\
\text { Discoloration } \\
\text { Calcification } \\
\text { Electric pulp test. (EPT) } \\
\text { Changes dimensions in Root } \\
\text { length (CDRL) } \\
\text { Changes in dimensions of the } \\
\text { dentin wall thickness at the } \\
\text { apical third of the root. (CDTA) } \\
\text { Changes in dimensions of the } \\
\text { dentin wall } \\
\text { thickness at the middle third of } \\
\text { the root (CDTM) } \\
\text { Changes in } \\
\text { dimensions of the dentin wall } \\
\text { thickness at the apical foramen } \\
\text { width (WF) }\end{array}$} & $\begin{array}{c}\text { CLS: } \\
\text { G1: } 100 \% \\
\text { G2: } 100 \%\end{array}$ \\
\hline & & & & & & & $\begin{array}{c}\text { IRL n (\%): } \\
\text { G1: } 21(100 \%) \\
\text { G2: } 22(100 \%)\end{array}$ \\
\hline & & & & & & & $\begin{array}{c}\text { Change in root length: } \\
\text { G1: } 16.40 \% \pm 13.6 \% \\
\text { G2: } 15.4 \% \pm 13.6 \% \\
(p>0.05)\end{array}$ \\
\hline & & & & & & & $\begin{array}{c}\text { IWTA: } \\
\text { G1:18 (86\%) } \\
\text { G2: } 20(91 \%)\end{array}$ \\
\hline & & & & & & & $\begin{array}{c}\text { IWTM: } \\
\text { G1: } 21(100 \%) \\
\text { G2: } 12(55 \%)\end{array}$ \\
\hline & & & & & & & $\begin{array}{c}\text { NAF: } \\
\text { G1: } 21(100 \%) \\
\text { G2: } 20(91 \%)\end{array}$ \\
\hline & & & & & & & $\begin{array}{l}\text { Discoloration: } \\
\text { G1: } 15(71 \%) \\
\text { G2: } 14(64 \%)\end{array}$ \\
\hline & & & & & & & $\begin{array}{l}\text { Calcification: } \\
\text { G1: } 10(48 \%) \\
\text { G2: } 12(55 \%)\end{array}$ \\
\hline
\end{tabular}


Table 1. Cont.

\begin{tabular}{|c|c|c|c|c|c|c|c|}
\hline Article & Patients Age/Sex & Intervention & Medication & $\begin{array}{c}\text { Preparation } \\
\text { of Scaffold }\end{array}$ & Restoration & Outcomes & Results \\
\hline & & & & & & & $\begin{array}{c}\text { EPT: } \\
\text { G1: } 7(33 \%) \\
\text { G2: } 4(18 \%) \\
\end{array}$ \\
\hline & & & & & & & $\begin{array}{c}\text { CDRL: } \\
\text { G1: } 16.4 \pm 13.6 \\
\text { G2: } 15.4 \pm 13.6 \\
\end{array}$ \\
\hline & & & & & & & $\begin{array}{c}\text { CDTA: } \\
\text { G1: } 21.5 \pm 22.5 \\
\text { G2: } 21.2 \pm 19.5 \\
\end{array}$ \\
\hline & & & & & & & $\begin{array}{c}\text { CDTM: } \\
\text { G1: } 23.8 \pm 21 \\
\text { G2: } 6.9 \pm 14 \\
\end{array}$ \\
\hline & & & & & & & $\begin{array}{c}\text { WF: } \\
\text { G1: }-65 \pm 34 \\
\text { G2: }-55 \pm 34 \\
\end{array}$ \\
\hline \multirow{2}{*}{$\begin{array}{l}\text { Santhakumar, } \\
\text { et al. [23] }\end{array}$} & \multirow{2}{*}{$\begin{array}{c}\text { Patients with } \\
\text { maxillary central } \\
\text { incisor immature } \\
\text { non-vital. } \\
(\mathrm{n}=40) \\
\text { Age: } 7-12 \text { years }{ }^{\dagger} .\end{array}$} & \multirow{2}{*}{$\begin{array}{l}\text { G1: PRF gel }(\mathrm{n}=20) \\
\text { G2: PRF membrane as } \\
\text { scaffold } \\
(\mathrm{n}=20)\end{array}$} & \multirow{2}{*}{$\begin{array}{c}\text { Irrig: } 3.0 \% \mathrm{NaCl} \text {. } \\
\text { ABX (MTZ, CPO, and } \\
\text { MNO) } \\
\text { TS: Intermediate } \\
\text { restorative materialt. } \\
\text { Time: } 3 \text { weeks }\end{array}$} & \multirow{2}{*}{$\begin{array}{l}\text { PRF by } 5 \mathrm{~mL} \text { of blood at } \\
3000 \mathrm{rpm} \text { for } \\
10 \text { min gel. } \\
\text { Membrane obtained by } \\
\text { PRF gel compression } t .\end{array}$} & \multirow{2}{*}{$\begin{array}{l}\text { MTA, type II glass } \\
\text { ionomer (Fugi 2) } \\
\text { cement, and } \\
\text { composite } \\
\text { material } \\
\text { (3 M ESPE). }\end{array}$} & \multirow{2}{*}{$\begin{array}{c}\text { Clinical success (CLS) } \\
\text { Radiographic } \\
\text { success (RCS) }\end{array}$} & $\begin{array}{l}\text { CLS: at } 18 \text { months } \\
\text { G1: positive } 18 \text {, negative } 1 \\
\text { G2: positive 18, negative } 1 \\
\quad(p=0.757)\end{array}$ \\
\hline & & & & & & & $\begin{array}{c}\text { RCS: at } 18 \text { months } \\
\text { G1: positive } 18 \text {, negative } 1 \\
\text { G2: positive 17, negative } 2 \\
(p=1.00)\end{array}$ \\
\hline \multirow[b]{2}{*}{$\begin{array}{l}\text { Ragab, } \\
\text { et al. [24] }\end{array}$} & \multirow[b]{2}{*}{$\begin{array}{c}\text { Patients with } \\
\text { maxillary central } \\
\text { incisor immature } \\
\text { non-vital. } \\
(\mathrm{n}=22) \\
\text { Age: } 7-12 \text { years } \\
\mathrm{M}(\mathrm{n}=32) \\
\mathrm{F}(\mathrm{n}=28)\end{array}$} & & \multirow[b]{2}{*}{$\begin{array}{l}\text { Irrig: } 5.0 \% \mathrm{NaCl} \text {. } \\
\text { ABX: (MTZ, CPO) } \\
\text { TS: Intermediate } \\
\text { restorative material, } \\
\text { glass ionomer } \\
\text { cement } \\
\text { (KetacCemEasymix, } \\
\text { 3M Deutschland } \\
\text { GmbH, Germany } \\
\text { Time: } 3 \text { weeks }\end{array}$} & & \multirow[b]{2}{*}{$\begin{array}{l}\text { MTA (Productos } \\
\text { Odontológicos S/A, } \\
\text { Brasil), glass ionomer } \\
\text { (SD I, Victoria, } \\
\text { Australia) }\end{array}$} & \multirow[b]{2}{*}{$\begin{array}{c}\text { Clinical success (CLS) } \\
\text { Increase in root length (IRL) } \\
\text { Apical and } \\
\text { cervical barriers calcific (ACBC) }\end{array}$} & $\begin{array}{c}\text { CLS: } \\
\text { G1:100\% } \\
\text { G2:100\% }\end{array}$ \\
\hline & & $\begin{array}{c}\text { G1: BC } \\
(\mathrm{n}=11) \\
\mathrm{G} 2: \mathrm{BC}+\mathrm{PRF}(\mathrm{n}=11)\end{array}$ & & $\begin{array}{l}\text { PRF by } 12 \mathrm{~mL} \text { of blood at } \\
3000 \mathrm{rpm} \text { for } 12 \mathrm{~min}\end{array}$ & & & $\begin{array}{c}\text { IRL: } \\
\text { G1: } 6 \text { months } 7.7 \%, \\
\text { 12 months } 14.8 \% . \\
\text { G2: } 6 \text { month } 8.8 \%, \\
\text { 12 months } 12.3 \% \\
\text { (no data of SD) } \\
\text { No statistically significant } \\
\text { difference between } \\
\text { the two groups }\end{array}$ \\
\hline
\end{tabular}


Table 1. Cont.

\begin{tabular}{|c|c|c|c|c|c|c|c|}
\hline Article & Patients Age/Sex & Intervention & Medication & $\begin{array}{l}\text { Preparation } \\
\text { of Scaffold }\end{array}$ & Restoration & Outcomes & Results \\
\hline & & & & & & & $\begin{array}{c}\text { ACBC: } \\
\text { G1: cervical } 4 \text { cases ( } 36.4 \%) ; \text { apical } 5 \\
\text { cases }(45.4 \%) ; \text { both cervical and } \\
\text { apical in the same tooth } 1 \text { case }(9 \%) ; \\
\text { no calcific barriers observed } 3 \text { cases } \\
\text { ( } 27 \%) . \\
\text { G2: cervical } 4 \text { cases }(36.4 \%) \text {; apical } 7 \\
\text { cases (63.6\%); both cervical and } \\
\text { apical in the same tooth } 3 \text { cases } \\
\text { (27\%); no calcific barriers } \\
\text { observed } 3 \text { cases }(27 \%) .\end{array}$ \\
\hline \multirow{6}{*}{$\begin{array}{l}\text { Ulusoy, } \\
\text { et al. [25] }\end{array}$} & \multirow{6}{*}{$\begin{array}{l}\text { Patients with incisor } \\
\text { immature non-vital. } \\
\text { ( } \mathrm{n}=88 \text { teeth) } \\
\text { Age: }(8-11)\end{array}$} & \multirow{6}{*}{$\begin{array}{l}\text { G1: PRP } \\
(\mathrm{n}=18) \\
\text { G2:PRF } \\
(\mathrm{n}=17) \\
\text { G3: PP } \\
(\mathrm{n}=17) \\
\text { G4: BC } \\
(\mathrm{n}=21)\end{array}$} & \multirow{6}{*}{$\begin{array}{l}\text { Irrig: } 1.25 \% \mathrm{NaCl} \\
\text { Metronidazole, } \\
\text { ciprofloxacin, } \\
\text { and clindamycin. } \\
\text { TS: } 4 \text { mm glass } \\
\text { ionomer cement } \\
\text { (Fuji IX; GC } \\
\text { America, Alsip, IL). } \\
\text { Time: } 4 \text { weeks }\end{array}$} & \multirow{6}{*}{$\begin{array}{c}\text { PRP: } 20 \mathrm{~mL} \text { of blood }+15 \\
\text { mL citrate solution at } 1250 \\
\text { rpm } \\
\text { for } 15 \text { min. } \\
\text { PRF: } 10 \mathrm{~mL} \text { blood at } 3000 \\
\text { rpm for } 10 \text { min without } \\
\text { anticoagulant } \\
\text { PP: PRP was obtained } \\
\text { without erythrocytes and } \\
\text { leukocytes as with the PRP } \\
\text { group, and then a second } \\
\text { centrifugation was } \\
\text { performed for } 10 \text { min at } \\
4000 \text { rpm. }\end{array}$} & \multirow{6}{*}{$\begin{array}{c}\text { MTA ProRoot } \\
\text { Dentsply Tulsa, glass } \\
\text { ionomer base, the final } \\
\text { resin restoration } \\
\text { (Filtek; 3 M ESPE, St } \\
\text { Paul, MN) }\end{array}$} & \multirow{6}{*}{$\begin{array}{c}\text { Clinical success (CLS) } \\
\text { Changes in Root Dimensions at } \\
\text { the Follow-up Time (CDRT) } \\
\text { Increase in root width (IRW) } \\
\text { Increase in root } \\
\text { length (IRL) } \\
\text { Increase in } \\
\text { root area (IRA) } \\
\text { Decrease in } \\
\text { canal area (DAC) }\end{array}$} & $\begin{array}{c}\text { CLS: (success rates 1 and 2) } \\
\text { G1: 18/18; G2: 16/17; G3: 17/17; G4: } \\
\text { 20/21 }\end{array}$ \\
\hline & & & & & & & CDRT: \%, 27 months \\
\hline & & & & & & & $\begin{array}{c}\text { IRW: (\%) } \\
\text { G1: } 19.48 \pm 3.62 \\
\text { G2: } 11.14 \pm 3.89 \\
\text { G3: } 9.99 \pm 3.92 \\
\text { G4: } 12.25 \pm 4.09(p>0.05) \\
\end{array}$ \\
\hline & & & & & & & $\begin{array}{c}\text { IRL: (\%) } \\
\text { G1: } 5.11 \pm 1.30 \\
\text { G2: } 7.05 \pm 1.39 \\
\text { G3: } 5.30 \pm 1.41 \\
\text { G4: } 5.06 \pm 1.47(p>0.05) \\
\end{array}$ \\
\hline & & & & & & & $\begin{array}{c}\text { IRA: }(\%) \\
\text { G1: } 10.25 \pm 2.25 \\
\text { G2: } 8.95 \pm 2.41 \\
\text { G3: } 8.70 \pm 2.43 \\
\text { G4: } 11.77 \pm 2.54(p>0.05)\end{array}$ \\
\hline & & & & & & & $\begin{array}{c}\text { DAC: (\%) } \\
\text { G1: }-15.03 \pm 5.25 \\
\text { G2: }-19.11 \pm 5.6 \\
\text { G3: }-12.57 \pm 5.68 \\
\text { G4: }-26.02 \pm 5.93(p>0.05)\end{array}$ \\
\hline
\end{tabular}


Table 1. Cont.

\begin{tabular}{|c|c|c|c|c|c|c|c|}
\hline Article & Patients Age/Sex & Intervention & Medication & $\begin{array}{l}\text { Preparation } \\
\text { of Scaffold }\end{array}$ & Restoration & Outcomes & Results \\
\hline \multirow{9}{*}{$\begin{array}{l}\text { Rizk et al. } \\
\text { (a) [26] }\end{array}$} & \multirow{9}{*}{$\begin{array}{l}\text { Patients with bilateral } \\
\text { maxillary immature } \\
\text { permanent central incisors } \\
\text { with necrotic pulp. } \\
\text { Patients: } \\
\text { Age: } 8-14 \text { years } \\
\text { M }(\mathrm{n}=7) \\
\mathrm{F}(\mathrm{n}=6) \\
\text { Teeth } \\
(\mathrm{n}=26)\end{array}$} & \multirow{9}{*}{$\begin{array}{l}\text { G1: PRPS } \\
(\mathrm{n}=13) \\
\text { G2: BC } \\
(\mathrm{n}=13)\end{array}$} & \multirow{9}{*}{$\begin{array}{c}\text { Irrig: } 2.0 \% \mathrm{NaCl} \\
\text { ABX (MTZ, CPO, and } \\
\text { MNO) } \\
\text { TS: Coltosol F } \\
\text { Time: } 3 \text { weeks }\end{array}$} & \multirow{9}{*}{$\begin{array}{l}\text { PRP by } 4.5 \mathrm{~mL} \text { blood } 2400 \\
\text { rpm for } 10 \mathrm{~min} \text { and } 3600 \\
\text { rpm } 15 \mathrm{~min} 0.5 \mathrm{~mL} 10 \% \\
\text { calcium chloride }+ \text { sterile } \\
\text { collagen sponge. }\end{array}$} & \multirow{9}{*}{$\begin{array}{l}\text { MTA and II glass } \\
\text { ionomer material (GC } \\
\text { America, Alsip, IL) }\end{array}$} & \multirow{9}{*}{$\begin{array}{c}\text { Clinical success (CLS) } \\
\text { Increase in root length (IRL) } \\
\text { Increase in Root Thickness (IRT) } \\
\text { Increase in Bone Density (IBD) } \\
\text { Decrease in apical diameter } \\
\text { (DAD) }\end{array}$} & $\begin{array}{c}\text { CLS: } \\
\text { G1: } 100 \% \\
\text { G2: } 100 \%\end{array}$ \\
\hline & & & & & & & $\begin{array}{c}\text { IRL: } \\
3 \text { months }(\mathrm{mm}, \%): \\
\text { G1: } 0.225 \pm 0.19(1.52 \% \pm 1.43 \%) \\
\text { G2: } 0.133 \pm 0.217(0.967 \% \pm 1.75 \%) \\
(p=0.006 *)\end{array}$ \\
\hline & & & & & & & $\begin{array}{c}6 \text { months }(\mathrm{mm}, \%): \\
\text { G1: } 0.557 \pm 0.23(3.7 \% \pm 1.43 \%) \\
\text { G2: } 0.273 \pm 0.29(1.92 \% \pm 2.32 \%) \\
(p=0.0028) .\end{array}$ \\
\hline & & & & & & & $\begin{array}{c}9 \text { months }(\mathrm{mm}, \%): \\
\text { G1: } 0.996 \pm 0.35(6.6 \% \pm 2.4 \%) \\
\text { G2: } 0.449 \pm 0.35(3.11 \% \pm 2.8 \%) \\
(p=0.001 \%) .\end{array}$ \\
\hline & & & & & & & $\begin{array}{c}12 \text { months }(\mathrm{mm}, \%): \\
\text { G1: } 1.48 \pm 0.37(9.88 \% \pm 2.85 \%) \\
\text { G2: } 0.68 \pm 0.44(4.68 \% \pm 3.45 \%) \\
(p=0.001 *)\end{array}$ \\
\hline & & & & & & & $\begin{array}{c}\text { IRT: } \\
3 \text { months }(\mathrm{mm}, \%): \\
\text { G1: } 0.153 \pm 0.128(6.03 \% \pm 5.03 \%) \\
\text { G2: } 0.133 \pm 0.27(5.45 \% \pm 9.48 \%) \\
(p=0.806)\end{array}$ \\
\hline & & & & & & & $\begin{array}{c}6 \text { months }(\mathrm{mm}, \%) \\
\text { G1: } 0.445 \pm 0.41(18.05 \% \pm 17.45 \%) \\
\text { G2: } 0.335 \pm 0.506(12.46 \% \pm 17.95 \%) \\
\left(p=0.019^{*}\right) .\end{array}$ \\
\hline & & & & & & & $\begin{array}{c}9 \text { months }(\mathrm{mm}, \%) \\
\text { G1: } 0.739 \pm 0.56(29.65 \% \pm 23.9 \%) \\
\text { G2: } 0.49 \pm 0.527(18.44 \% \pm 19.95 \%) \\
(p=0.003 *)\end{array}$ \\
\hline & & & & & & & $\begin{array}{c}12 \text { months }(\mathrm{mm}, \%): \\
\text { G1: } 0.97 \pm 0.75(39.27 \% \pm 32.04 \%) \\
\text { G2: } 0.68 \pm 0.678(25.56 \% \pm 26.5 \%) \\
(p=0.002 *)\end{array}$ \\
\hline
\end{tabular}


Table 1. Cont. $(p=0.027)$.

6 months (grey value, \%):

$34.42 \pm 21.03(42.3 \% \pm 25.46 \%)$ G2: $33.8 \pm 17.87$

$(0.355 \% \pm 0.235 \%),(p=0.027 *)$.

9 months (grey value, \%):

G1: $52.47 \pm 25.39$

G2: $44.91 \pm 21.01$

$(0.468 \% \pm 0.283 \%),(p=0.027)$.

12 months (grey value, \%):

G1: $65.08 \pm 30.043(71.84 \% \pm$

$(0.609 \%+0.27 \%),(p=0.027)$

$$
\text { DAD: }
$$

3 months (mm,

G1: $0.25 \pm 0.167(9.91 \% \pm 6.03 \%)$

G2: $0.137 \pm 0.063(6.06 \% \pm 3.7 \%),(p$ $=0.008^{*}$ ).

6 months ( $\mathrm{mm}, \%$ )

G1: $0.656 \pm 0.43(27.29 \% \pm 14.1 \%)$

G2: $0.42 \pm 0.27(18.24 \% \pm 11.11 \%)$,

9 months $\mathrm{mm}(\%)$

G1: $2.17 \pm 3.86(51.98 \% \pm 19.64 \%)$, $\pm 3.9(40.7 \% \pm$
$(p=0.002 *)$.

12 months $\mathrm{mm}(\%):$
G1: $2.49+3.93(64.83 \%+18.5 \%)$

G1: $2.49 \pm 3.93(64.83 \% \pm 18.5 \%)$,
G2: $2.2 \pm 3.97(53.45 \% \pm 19.4 \%)$ $(p=0.003 *)$. 
Table 1. Cont.

\begin{tabular}{|c|c|c|c|c|c|c|c|}
\hline Article & Patients Age/Sex & Intervention & Medication & $\begin{array}{c}\text { Preparation } \\
\text { of Scaffold }\end{array}$ & Restoration & Outcomes & Results \\
\hline \multirow{3}{*}{$\begin{array}{c}\text { ElSheshtawy et al. } \\
{[27]}\end{array}$} & \multirow{3}{*}{$\begin{array}{l}\text { Patients with } \\
\text { immature permanent } \\
\text { anterior teeth with } \\
\text { necrotic pulps }\end{array}$} & \multirow{3}{*}{$\begin{array}{l}\text { G1: PRP } \\
\text { G2: BC }\end{array}$} & \multirow{3}{*}{$\begin{array}{l}\text { Irrig: } 5.25 \% \mathrm{NaCl} \\
\text { Metronidazole, } \\
\text { ciprofloxacin, } \\
\text { and minocycline. } \\
\text { TS: Coltosol F }\end{array}$} & \multirow{3}{*}{$\begin{array}{l}\text { PRP: blood } 2400 \text { rpm for } 10 \\
\text { min and } 3600 \text { rpm for } 15 \\
\text { min }\end{array}$} & \multirow{3}{*}{$\begin{array}{l}\text { MTA, glass } \\
\text { ionomer (RIVA } \\
\text { self-cure, } \\
\text { SDI limited, } \\
\text { Bayswater, } \\
\text { Victoria, } \\
\text { Australia) and resin } \\
\text { composite (Filtek } \\
\text { z250 universal } \\
\text { restorative, 3 mol L1 } \\
\text { ESPE, St. Paul, MN, } \\
\text { USA). }\end{array}$} & \multirow{3}{*}{$\begin{array}{c}\text { Clinical Success } \\
\text { (CLS) } \\
\text { Pulp sensibility (thermal } \\
\text { electrical) } \\
\text { Root length } \\
\text { (RL) } \\
\text { Dentinal wall thickness } \\
\text { (RDT) } \\
\text { Apical } \\
\text { foramen width } \\
\text { (AFW) } \\
\text { Radiographic } \\
\text { root area } \\
\text { (RRA) } \\
\text { Periapical area } \\
\text { diameters (PAD) }\end{array}$} & $\begin{array}{c}\text { CLS: } \\
6 \text { months: G1: } 12 / 14(85.7 \%) ; \mathrm{G} 2: \\
15 / 17(88 \%)\end{array}$ \\
\hline & & & & & & & $\begin{array}{c}\text { Pulp sensibility: } \\
\text { 12 months: } \\
\text { G1: } 100 \% \text { lack of response } \\
\text { G2: } 100 \% \text { lack of response } \\
\end{array}$ \\
\hline & & & & & & & $\begin{array}{c}\text { Time changes (time effect) in RL, } \\
\text { RDT, AFW, RRA, and PAD were } \\
\text { found to be significant }(\mathrm{P}<0.001) \text {. } \\
\text { No difference between the REP. No } \\
\text { more data. }\end{array}$ \\
\hline \multirow{8}{*}{$\begin{array}{l}\text { Rizk et al. } \\
\text { (b) [28] }\end{array}$} & \multirow{8}{*}{$\begin{array}{c}\text { Pediatric Patients with } \\
\text { permanent maxillary } \\
\text { central incisors } \\
\text { immature } \\
\text { and necrosis. } \\
\text { Patients } \\
\text { Age: } 8-14 \text { year } \\
\mathrm{M}(\mathrm{n}=7) \\
\mathrm{F}(\mathrm{n}=6) \\
\text { Teeth } \\
(\mathrm{n}=26)\end{array}$} & \multirow{8}{*}{$\begin{array}{l}\text { G1: PRPS } \\
(\mathrm{n}=13) \\
\text { G2: PRFS } \\
(\mathrm{n}=13)\end{array}$} & \multirow{8}{*}{$\begin{array}{c}\text { Irrig: } 2.0 \% \mathrm{NaCl} \\
\text { ABX (MTZ, CPO and } \\
\text { MNO) } \\
\text { TS: Coltosol F } \\
\text { Time: } 3 \text { weeks }\end{array}$} & \multirow{8}{*}{$\begin{array}{c}\text { PRPS: blood } 2400 \mathrm{rpm} \text { for } \\
10 \mathrm{~min} \text { and } 3600 \mathrm{rpm} \text { for } 15 \\
\mathrm{~min}+2 \times 2 \mathrm{~mL} \text { of sterile } \\
\text { collagen sponge. } \\
\text { PRFS: blood at } 3000 \mathrm{rpm} \\
\text { for } 10 \text { min }+2 \times 2 \mathrm{~mL} \text { of } \\
\text { sterile } \\
\text { collagen sponge. }\end{array}$} & \multirow{8}{*}{$\begin{array}{c}\text { Glass ionomer (GC } \\
\text { America, 213 Alsip, IL) } \\
\text { and composite (Z 250, } \\
\text { 3 M ESPE). }\end{array}$} & \multirow{8}{*}{$\begin{array}{l}\text { Increase in root length (IRL) } \\
\text { Increase in root width (WF) } \\
\text { Increase in bone density in grey } \\
\text { (IBD) } \\
\text { Decrease in apical diameter } \\
\text { (DAD) }\end{array}$} & $\begin{array}{c}\text { CLS: } \\
\text { G1: } 13 / 13 \\
\text { G2: } 13 / 13\end{array}$ \\
\hline & & & & & & & IRL: \\
\hline & & & & & & & $\begin{array}{c}3 \text { months }(\mathrm{mm}, \%): \\
\text { G1: } 0.225 \pm 0.19(1.52 \% \pm 1.43 \%) \\
\text { G2: } 0.155 \pm 0.099(1.02 \% \pm 0.673 \%) \\
(p=0.406)\end{array}$ \\
\hline & & & & & & & $\begin{array}{c}6 \text { months }(\mathrm{mm}, \%): \\
\text { G1: } 0.557 \pm 0.23(3.7 \% \pm 1.43 \%) \\
\text { G2: } 0.391 \pm 0.187(2.57 \% \pm 1.23 \%) \\
(p=0.127)\end{array}$ \\
\hline & & & & & & & $\begin{array}{c}9 \text { months }(\mathrm{mm}, \%) \\
\text { G1: } 0.996 \pm 0.35(6.6 \% \pm 2.4 \%) \\
\text { G2: } 0.793 \pm 0.378(5.2 \% \pm 2.48 \%) \\
(p=0.174)\end{array}$ \\
\hline & & & & & & & $\begin{array}{c}12 \text { months }(\mathrm{mm}, \%): \\
\text { G1: } 1.48 \pm 0.37(9.88 \% \pm 2.85 \%) \\
\text { G2: } 1.24 \pm 0.54(8.19 \% \pm 3.64 \%) \\
(p=0.355) \\
\end{array}$ \\
\hline & & & & & & & WF: \\
\hline & & & & & & & $\begin{array}{c}3 \text { months }(\mathrm{mm}, \%): \\
\text { G1: } 0.153 \pm 0.128(6.03 \% \pm 5.03 \%) \\
\text { G2: } 0.19494 \pm 0.172(7.9 \% \pm 6.2 \%) \\
(p=0.47)\end{array}$ \\
\hline
\end{tabular}


Table 1. Cont.

9 months ( $\mathrm{mm}, \%)$ : G2: $0.73517 \pm 0.34$ $(30.77 \% \pm 13.26 \%)(p=0.503)$

12 months (mm, \%):

G1: $0.97 \pm 0.75(39.27 \% \pm 32.04 \%)$ $(42.37 \% \pm 16.49 \%)(p=0.574)$ IBD

3 months (grey value, \%): G1: $24.87 \pm 16.63$ $(27.605 \% \pm 19.89 \%)$

G2:15.64 $\pm 11.1(45.14 \% \pm 74.78 \%)$ $(p=0.345)$

6 months (grey value, \%):

G1: $34.42 \pm 21.03(42.3 \% \pm 25.46 \%)$ $(p=0.345)$

9 months (grey value, $\%$ ) G1: $52.47 \pm 25.39$

G2: $40.79 \pm 19.12(109.29 \% \pm$

$171.22 \%)(p=0.414)$

12 months (grey value, \%): G1: $65.08 \pm 30.043$ $(71.84 \% \pm 30.043 \%)$ G2: $53.44 \pm 22.165$ $(p=0.345)$
$(137.4 \%$
$(2)$ DAD:

$$
\text { DAD: }
$$

3 months ( $\mathrm{mm}, \%)$

G1: $0.25 \pm 0.167(9.91 \% \pm 6.03 \%)$ G2: $0.34 \pm 0.2(15.7 \% \pm 8.84 \%)$ $(p=0.246)$ 6 months (mm, \%)

G1: $0.656 \pm 0.43(27.29 \% \pm 14.1 \%)$ G2: $0.87 \pm 0.48(38.23 \% \pm 15.03 \%)(p$ $=0.123)$ 
Table 1. Cont.

Article

Intervention

Medication
Preparation
of Scaffold
Restoration

Outcomes

Results

9 months $(\mathrm{mm}, \%)$

G2: $1.33 \pm 0.57(58.89 \% \pm 10.59 \%)(p$ $=0.611$

12 months $(\mathrm{mm}, \%)$

G1: $2.49 \pm 3.93(64.83 \% \pm 18.5 \%)$ $(p=0.437)$

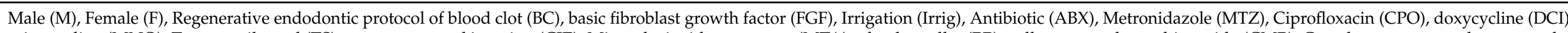

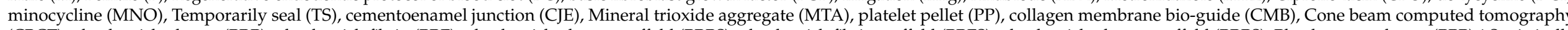

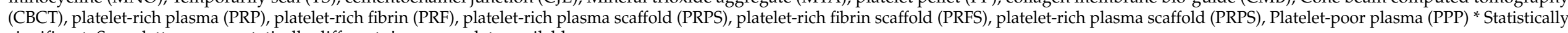

significant. Same letters are no statically different, $\uparrow$ no more data available. 
Nagy et al. [20] evaluated (at 18 months) the assessment of pain and/or swelling after the use of BC plus a gelatin hydrogel incorporating FGF for REP. At the first visit, the participants included in the study were anesthetized using local anesthesia without a vasoconstrictor and underwent conventional cavity preparation with instrumentation technique, sodium hypochlorite washes, placement of triple antibiotic paste (metronidazole, ciprofloxacin, and doxycycline), and temporary sealing with Coltosol F (Zinc Oxide/Zinc Sulphate). Later, the participants were distributed into three groups. The first group was treated with a mineral trioxide aggregate (MTA) apical plug (G1), the second group was treated with REP using protocol of BC (G2), and the third group was treated with BC plus FGF. The researchers reported success rates of $100 \%, 90 \%$, and $80 \%$ (G1, G2, and G3, respectively).

Alagl et al. [1] used a triple antibiotic paste (ciprofloxacin, metronidazole, and cefaclor) in each tooth for disinfection of the root canal, and subsequently, they used a tissue scaffold using PRP (G1) or BC (G2) and cover them with the addition of MTA, carried out a 12-month follow-up evaluated the clinical success, the researchers reported that, from 1 to 12 months of follow-up, both groups showed a resolution of signs and symptoms (pain, swelling, fistula, and/or sensitivity to percussion and palpation) in $100 \%$ of the treated teeth.

Shivashankar et al. [21] evaluated at 12 months the assessment of pain, inflammation, or swelling after the use of PRF or PRP for REP. The patients in the first visit underwent conventional cavity preparation and instrumentation, sodium hypochlorite washes, placement of triple antibiotic paste, and the access to the cavity was closed with cotton pellets and intermediate restorative material. After 3 weeks, the participants were distributed in the group of PRF (G1), or the group of BC (G2), or the group of PRP (G3). The researchers reported success rates of $90 \%, 100 \%$, and $100 \%$ (G1, G2, and G3, respectively).

Jiang et al. [22] evaluated (for at least 6 months) the use of the CMB for REP. The participants, in a first visit, underwent the conventional cavity preparation with instrumentation technique, sodium hypochlorite washes, placement of calcium hydroxide paste, and temporary sealing with glass ionomer. Later, the participants were distributed into the group of intervention (G1) treated by $\mathrm{BC}+\mathrm{CMB}$ or the control group (G2) using only BC. The researchers reported that both study groups were clinically successful for achieve the complete resolution of signs and symptoms. However, in this study, there was a lack of the criteria, signs, and symptoms considered for determining the periapical healing.

Santhakumar et al. [23] conducted a triple-blind clinical trial to evaluate the use of PRF in two modalities as biological scaffolds in REP. These preparations were platelet-rich fibrin in its gel form (PRFg) and membrane form (PRFm). The study group compared the clinical success (absence of pain, swelling, mobility, and lack of sensitivity to percussion). The research group reported that, after 6 and 12 months of the treatment, both groups were clinically asymptomatic. Also, after 18 months of follow-up in both groups, a failed case with mobility teeth and pain were shown, concluding a similar success for both groups.

Ragab et al. [24] evaluated (at 12 months) the clinical success based on the regression of signs and symptoms associated with necrotic teeth in two groups of REP. The study group, treated with $\mathrm{BC}$ plus PRF, was compared with a group treated with $\mathrm{BC}$. The authors reported that all cases showed $100 \%$ success in both groups.

Ulusoy et al. [25] evaluated success rates every 3 months with a follow-up period of $28.25 \pm 1.20$ months in four groups of REP following according to the treatment used: PRP group (G1), PRF group (G2), platelet pellet group (G3), or BC group (G4). The success rates were scored as 0 (failure), 1 (resolution of disease), or 2 (resolution of disease + radiographic root development and positive response to cold testing and electric pulp testing). The authors reported that in all groups, the most commonly rated success score was $2(G 1=11 / 18$, $\mathrm{G} 2=11 / 17, \mathrm{G} 3=13 / 17, \mathrm{G} 4=15 / 21)$, and there was no significant difference in the success scores among groups.

Rizk et al. (a) [26] compared, clinically and radiographically, the regenerative potential of PRP (G1) compared to BC (G2), and concluded (about the primary clinical outcomes of 
resolution of pain, swelling, mobility, and sinus/fistula) that all cases showed $100 \%$ success. At 12 months of follow-up, at the end of the study, all revascularized/revitalized teeth did not respond to the pulp sensitivity tests, thermal (cold/heat), and electrical test, with no statistically significant differences between the two groups.

ElSheshtawy et al. [27] evaluated the clinical success in teeth treated with PRP (G1) compared to BC (G2) for REP. The clinical examination was performed by assessing tooth color, tenderness to percussion, swelling, sinus tracts, and mobility. At 6 months of followup, the authors reported around $85 \%$ of success in both groups.

Rizk et al. (b) [28] evaluated the regenerative potential of a platelet-rich fibrin scaffold (PRPS) (G1) versus platelet-rich fibrin scaffold (PRFS) (G2) for the treatment of the NIT (upper central incisors). The PRPS was prepared according to the method of Dohan and Choukroun and subsequently, a $2 \times 2 \mathrm{~mm}$ of sterile collagen sponge soaked in PRPS was introduced into the root canal at the level of the cement-enamel junction. On the other hand, the PRFS obtained was cut into small fragments using a scalpel blade and was placed incrementally inside the canal using appropriate endodontic plugs up to the level of the cement-enamel junction, subsequently, $2 \times 2-\mathrm{mm}$ of sterile collagen sponge was introduced on top of the PRFS (G2). They reported that no statistically significant differences were found between the two groups regarding the primary clinical outcomes (resolution of pain, swelling, mobility, and sinusitis/fistula). All the cases showed $100 \%$ success and all the revascularized/revitalized teeth in the present study had no response to the sensitivity tests, thermal (cold/heat), and electrical test during the entire follow-up of 12 months.

The certainty in evidence from eight studies comparing the clinical success of the experimental REP versus BC was low as summarized in Table 2. The results of the meta-analysis in the present review suggested that clinical success showed no differences between the patients who received REP with any type of experimental scaffolds or matrix in comparison with those that receive BC, as shown in Figure 3.

\subsection{Root Length}

Radiographic evidence showed an increase in root length (IRL) and the thickness of the root wall as well as the formation of a radiographic apex. These results are desirable, although not always achievable, and neither are essential to accomplish a successful treatment.

Nagy et al. [20] evaluated, by radiographs, the IRL for the regeneration treatment of immature permanent teeth with necrotic pulps with a follow-up at 3, 6, 12, and 18 months in three treatment groups: group 1 (G1) with Mineral trioxide aggregate plug (MTA), group 2 (G2) with BC, and group 3 (G3) using BC plus a hydrogel scaffold with $150 \mu \mathrm{g}$ fibroblast basic growth factor (FGF). The research team reported that groups 2 and 3 showed no significant differences regarding the IRL during the entire follow-up period. Jiang et al. [22] compared dentin formation between an experimental group (G1) in which a Bio-Gide collagen membrane (CMB) served as a scaffold and a control group (G2) without the membrane (SMB). The patients were evaluated at intervals of 3 months and up to at least 6 months by means of radiographic evaluation a slightly greater change in the length of the root was determined in the experimental group; however, no statistically significant differences were found (Mann-Whitney $U$ test, $p>0.05$ ).

Alagl et al. [1] measured the length of each tooth from the most apical point of the root to the enamel cementum junction by cone-beam computed tomography in PRP (G1) and BC (G2) for REP. The research team reported in the follow-up of 12 months a statistically significant mean difference in root length in favor of PRP $(p<0.004)$. Contrarily, Shivashankar et al. [21] evaluated, at 12 months, the root lengthening and the lateral wall thickening using a Shei's ruler and categorized this outcome into satisfactory or good if there was an increase in a single increment and two increments, respectively. They reported that, at the end of 12 months, according to the chi-square test, there was no significant difference among the three groups (PRF, PRP, and BC). 
Table 2. Quality assessment of the included studies in the systematic review.

\begin{tabular}{|c|c|c|c|c|c|c|c|c|c|c|c|c|}
\hline \multicolumn{7}{|c|}{ Certainty Assessment } & \multicolumn{2}{|c|}{ No of Patients } & \multicolumn{2}{|r|}{ Effect } & \multirow[t]{2}{*}{ Certainty } & \multirow[t]{2}{*}{ Importance } \\
\hline $\begin{array}{c}\text { No of } \\
\text { Studies/Outcome }\end{array}$ & Study Design & $\begin{array}{c}\text { Risk } \\
\text { of Bias }\end{array}$ & Inconsistency & Indirectness & Imprecision & $\begin{array}{c}\text { Other } \\
\text { Considerations }\end{array}$ & $\begin{array}{c}\text { Experimental } \\
\text { REP }\end{array}$ & BC & $\begin{array}{l}\text { Relative } \\
(95 \% \text { CI })\end{array}$ & $\begin{array}{l}\text { Absolute } \\
(95 \% \text { CI })\end{array}$ & & \\
\hline \multicolumn{13}{|c|}{ Clinical success } \\
\hline \multicolumn{13}{|c|}{ IRL $\%$ at 12 months } \\
\hline 5 & $\begin{array}{l}\text { randomized } \\
\text { trials }\end{array}$ & serious & serious & not serious & not serious & none & 79 & 82 & - & $\begin{array}{c}\text { MD } 2.62 \text { higher } \\
(0.24 \text { lower to } 5.48 \\
\text { higher })\end{array}$ & $\oplus \oplus \circ \bigcirc$ LOW & IMPORTANT \\
\hline
\end{tabular}

REP: Regenerative endodontic procedure; BC: Blood clot; CI: Confidence interval; RR: Risk ratio; IRL: Increase in Root Length; MD: Mean difference. 


\begin{tabular}{|c|c|c|c|c|c|c|c|c|c|c|c|}
\hline \multirow[b]{2}{*}{ Study or Subgroup } & \multicolumn{2}{|c|}{ Intervention } & \multicolumn{2}{|c|}{ Control } & \multirow[b]{2}{*}{ Weight } & \multirow{2}{*}{\multicolumn{2}{|c|}{$\begin{array}{c}\text { Risk Ratio } \\
\text { M-H, Random, } 95 \% \mathrm{Cl}\end{array}$}} & \multirow{2}{*}{\multicolumn{2}{|c|}{$\begin{array}{c}\text { Risk Ratio } \\
\text { M-H, Random, } 95 \% \mathrm{Cl} \\
\end{array}$}} & & \\
\hline & Events & Total & Events & Total & & & & & & & \\
\hline Alagl, et al. & 15 & 15 & 15 & 15 & $16.0 \%$ & $1.00[0.88,1.13]$ & & & & & \\
\hline ElSheshtawy, et al. & 12 & 14 & 15 & 17 & $3.3 \%$ & $0.97[0.74,1.28]$ & & & & & \\
\hline Jiang, et al. & 23 & 23 & 23 & 23 & $36.3 \%$ & $1.00[0.92,1.09]$ & & & & & \\
\hline Nagy, et al. & 10 & 12 & 11 & 12 & $2.7 \%$ & $0.91[0.67,1.23]$ & & & & & \\
\hline Ragab, et al & 11 & 11 & 11 & 11 & $8.9 \%$ & $1.00[0.85,1.18]$ & & & & & \\
\hline Rizk, et al. (a) & 13 & 13 & 13 & 13 & $12.2 \%$ & $1.00[0.87,1.15]$ & & & & & \\
\hline Shivashankar, et al. & 18 & 20 & 15 & 15 & $7.6 \%$ & $0.91[0.76,1.09]$ & & & - & & \\
\hline Ulusoy, et al. & 17 & 17 & 20 & 21 & $13.1 \%$ & $1.04[0.91,1.20]$ & & & & & \\
\hline Total $(95 \% \mathrm{Cl})$ & & 125 & & 127 & $100.0 \%$ & $0.99[0.95,1.05]$ & & & & & \\
\hline Total events & 119 & & 123 & & & & & & & & \\
\hline $\begin{array}{l}\text { Heterogeneity: Tau } \\
\text { Test for overall effect }\end{array}$ & $\begin{array}{l}0.00 ; \text { Chi } \\
Z=0.20\end{array}$ & $\begin{array}{l}=2.05 \\
=0.84\end{array}$ & $d f=7(P$ & $=0.96)$ & ;) $1^{2}=0 \%$ & & 0.5 & 0.7 & Control & 1.5 & $\sqrt{2}$ \\
\hline
\end{tabular}

Figure 3. Meta-analysis of the clinical success for regenerative endodontics procedures.

Santhakumar et al. [23] considered (as a radiographic success) the accomplishment of both criteria; the IRL and root-end closure (using a Shei's ruler). They reported that, at the end of 6 months, there was no radiographic success in the PRFg or PRFm groups. At 12 months, the evaluation suggested that the PRFg group was probably faster in achieving radiographic success; however, there was no statistical difference between groups $(p=0.89)$. After 18 months, an adequate crown-to-root ratio was achieved in 18 of 19 cases for the PRFg group and in 17 of 19 cases for the PRFm group.

Ragab et al. [24] evaluated (at 6 and 12 months) the IRL of non-vital immature maxillary central incisors after the treatment using PRF plus BC or BC alone; the measurement was performed using an imported radiographic image with Digora software. The authors reported that both groups showed the same IRL rate with no statistically significant difference between the two groups during the follow-up period.

Ulusoy et al. [25] performed a radiographic evaluation of IRL by two assessors (kappa coeficients $=0.85$ and 0.93 ) after the REP using PRF, PRP, PP, or BC. The image analysis was done with ImageJ software. The authors reported that all the groups showed no statistically significant differences. On the other hand, Rizk et al. (a) [26] performed a 12-month radiographic evaluation of the upper and immature bilateral permanent central incisors with pulp necrosis after the treatment using PRP scaffold compared to BC. They reported that the mean of the IRL (in $\mathrm{mm}$ or percentage) for the PRP scaffold group was greater compared to the $\mathrm{BC}$ group for all time points.

ElSheshtawy et al. [27] evaluated the IRL in teeth treated with PRP (G1) compared to BC (G2) for REP. The measurements were performed using radiographs and Cone-Beam Computed Tomography images by two assessors (inter-rater agreement $=0.999$ ), the time effect in IRL was significant, however, there were no differences between the G1 and G2.

Finally, Rizk et al. (b) [28] evaluated the regenerative potential of PRPS versus PRFS after following a root canal disinfection protocol for 21 days in NIT (central incisors). As a result of this research, a statistical difference was reported in the IRL in favor of PRPS at all evaluated time intervals.

The certainty in evidence from five studies comparing the IRL of the experimental REP versus BC was low as summarized in Table 2 . The results of the meta-analysis in the present review suggested that the IRL showed no differences between the patients who received REP with any experimental scaffolds or matrix compared to those that only receive REP, as can be seen in Figure 4. The funnel plot is not shown due to the small number of studies included in the meta-analysis.

\subsection{Changes in Apical Diameter}

In the present systematic review, six articles evaluated apical closure. In all of them, it was reported that there was a decrease in apical closure with the REP used in each study. Nagy et al. [20] calculated the difference in apical diameter before and after the intervention, using a pre-established measurement in millimeters. The statistical analysis did not show 
any significant difference between the BC and FGF groups at 3, 6, 12, and 18 months. Both groups had a significant difference at 12 and 18 months compared to the MTA group. Alagl et al. [1] evaluated the complete apical closure at 12 months in PRP (14/15) and BC (8/15). Jiang et al. [22] determined (through the radiographic evaluation) the width of the apical foramen and found no differences between the $\mathrm{CMB}$ experimental group and $\mathrm{BC}$ control group at 6 months. Santhakumar et al. [23] determined (by the radiographic assessment) the apical obliteration at 18 months, and this was achieved with both the PRFg (in 18/19 cases) and the PRFm (in 17/19 cases) groups, without statistical difference between the groups. ( $p=0.89$ ). In contrast, Rizk et al. (a). [26] evaluated the decrease in apical diameter and reported that, in the PRP group, there was a greater reduction in diameter in comparison with the $\mathrm{BC}$ group at the follow-up intervals. Likewise, Rizk et al. (b). [28] determined the decrease in apical diameter in the PRPS and PRFS groups during four evaluation periods and found statistically significant differences in favor of the PRP scaffold group.

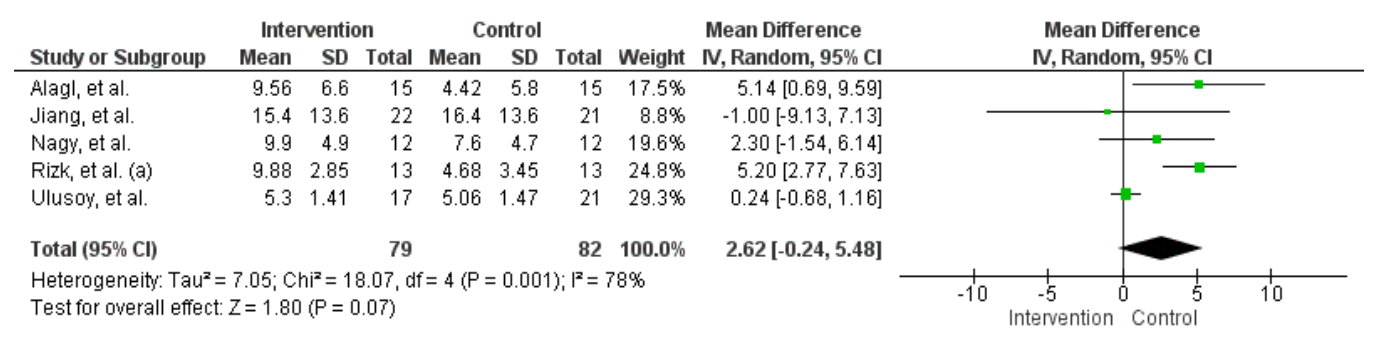

Figure 4. Meta-analysis of the root length for regenerative endodontics procedures.

\subsection{Dentin Thickness}

Five of the included studies evaluated the effect of FGF [20], CMB [22], PRF [21], PRP [21], or PRPS [26] to increase dentin thickness using BC as a control. Nagy et al. [20] reported that there was no statistical difference between the study groups. On the other hand, Jiang et al. [22] found significant differences in the first third of the root in favor of CMB. Shivashankar et al. reported no significant difference among PRF, PRP, and BC at the end of 12 months concerning root lateral wall thickness. As well as the reports of ElSheshtawy et al. [27]. Besides, Rizk et al. (a) [26] identified that PRP significantly increases root thickness compared to BC. However, Rizk et al. (b). [28] determined the effect of PRPS and PRFS and reported that there were no statistical differences between these groups.

\subsection{Bone Density}

In three of the included articles, the effect of FGF [20], PRP [1], or PRPS [26] was evaluated to increase bone density using BC as a control. Nagy et al. [20] and Alagl et al. [1] report that there was no statistical difference between the study groups. Contrarily, Rizk et al. (a). [26] identified that the use of PRPS significantly increases bone density compared to BC. On the other hand, Rizk et al. (b). [28] determined the effect of PRPS and PRFS, and report that there are no statistical differences between groups.

\subsection{Pulp Sensibility (Vital Test)}

In this review, seven studies evaluated the vitality of teeth in patients treated for REP using sensitivity tests (cold and/or electrical). Alagl et al. [1] showed a big vital responses reporting 18/30 teeth with positive responses (PRP $=80 \%, B C=40 \%$ ). Followed by Ulusoy et al. [25] who showed a positive response in the groups PRP $=61.11 \%$, $\mathrm{PRF}=64.7 \%, \mathrm{PP}=76.47 \%, \mathrm{BC}=71.42 \%$. However, Shivashankar et al. [21] reported $\mathrm{PRF}=15 \%, \mathrm{BC}=13.3 \%$ and $\mathrm{PRP}=15.8 \%$, moreover, Jiang et al. [22] reported $\mathrm{BC}+\mathrm{CBM}=18 \%$ and $\mathrm{BC}=33 \%$. Contrary, Rizk et al. (a) [26], ElSheshtawy et al. [27], and Rizk et al. (b) [28] reported a $100 \%$ lack of response in the studied groups (PRPS vs. BC, PRP vs. BC, and PRSF vs. PRFS, respectively). 


\section{Discussion}

Apexification using $\mathrm{Ca}(\mathrm{OH})_{2}$ or MTA is the standard treatment for NITs. However, with this technique, the treated tooth loses pulp vitality and various unfavorable consequences occur, such as the root development being interrupted. Therefore, the tooth remains with a weak root structure (an inadequate crown-to-root ratio and a high risk of fracture in the cervical root region due to functional stress) [29], which, in the long term, leads to dental extraction [30]. As an alternative option, REP has been proposed because it allows root development of these teeth. As in other endodontic procedures, disinfection is essential for the success of the procedure, which is why irrigants and intracanal antimicrobial agents are used. After that, intentional apical bleeding (BC) is induced to form a BC that serves as a cellular scaffold that promotes adequate apical healing.

The $\mathrm{BC}$ for apical revascularization has been accepted worldwide and several reviews have shown that this therapy improves clinical success rates (97.3-93.0\%). However, there is low consistency in favoring the rates of root elongation $(16.1 \%)$ and the increase in root thickness (39.8\%) [31]. Because of this, the postoperative root development in the NIT still represents a challenge and more efficient scaffold options have been developed. Alternative processes to $\mathrm{BC}$ for REP include the use of PRP and PRF. PRP is a derivative that is extracted from autologous blood, which contains three to eight times the concentration of platelets obtained by centrifugation and separation by density gradient. PRP contains several growth factors; hence its usage has been proposed in tissue regeneration [32]. Particularly in dentistry, PRP is used in the treatment of temporomandibular disorders [33], in oral and maxillofacial surgery [34], as well as in REP.

On the other hand, PRF possesses a tetra-molecular structure that serves as a natural autologous scaffold; it is obtained as a secondary platelet concentrate composed of cells such as leukocytes, stem cells, and platelets, in addition to soluble mediators such as interleukins. This natural scaffold supports micro-vascularization and works as a transport for carrying and differentiating macrophages M2, essentials for tissue regeneration and revascularization [35]. This PRF has mechanical properties and remodeling properties, which resemble the BC. However, there is insufficient information about the effectiveness of PRF and PRP in NIT; most of the research regarding the topic are case reports, case series [36,37], and just a few clinical trials with a small sample. Murray et al. reported in a systematic review and meta-analysis that the mean success rate for apical reduction or closure was $85.1 \%$ for PRP, $85.2 \%$ for PRF, and $58.8 \%$ for BC with a follow-up of one year. On the other hand, the mean success rate for root lengthening was $64.1 \%$ for $\mathrm{BC}, 64.2 \%$ for PRP, and $74.1 \%$ for PRF. Besides, dentinal wall thickening was 100\% for BC, $100 \%$ for PRP, and $100 \%$ for PRF. The majority of effects were similarly effective between the treatments (Fisher's exact test, $p>0.05$ ) except for the apical closure that occurred more frequently following PRP and PRF compared to BC (Fisher's exact test, $p<0.0011$ ) [38]. However, there is a lack of external validity in the meta-analysis. One important point is that in the included studies was that the populations are not sufficiently representatives. Further, the report does not include the impact of each study in the overall estimate, neither does the heterogeneity test with $\mathrm{I}^{2}$. There is not an analysis of the demography, treatments, or other methodological aspects that could act in favor of the intervention. Further, most of the included studies are case reports or case series that exhibited a lack of internal validity. In the present review, it was possible to carry out the meta-analysis in the clinical success and IRL outcomes because the other outcomes exhibited very heterogeneous methodologies and units of measurement that prevent pooled data.

The studies included in this systematic review related to the use of PRP or PRF lacks in to demonstrate the improvement of the clinical success or the increase of root length over BC in NIT. Besides, there is a lack of consistency in the vitality test. One expounds is that, despite stimulating tissue repair and repopulating the root canals of immature teeth, the new vital tissue may not possess the functional activity of the native pulp tissue [39].

The Bio-Gide is a biodegradable membrane compounded by I and III collagen types. It is used widely as a scaffold material in periodontal tissue regeneration [40]. In vivo 
experiments have shown that collagen type 1 can promote dental pulp cells (DPCs) differentiation into the odontoblast and embedded an osteodentin-like hard tissue matrix in the root canal [41]. Further, collagen membranes can promote migration, proliferation, and differentiation of mesenchymal stem cells [42]. These characteristics provide advantages such as stabilizing BC, maintaining growth factor levels, and promoting tissue regeneration. There was only one RTC included in the review related to collagen scaffold for REP and it does not demonstrate the improvement of the clinical success or the increase of root length over BC.

REP success depends on the regenerative proprieties of periradicular tissues and their capacity relates to the presence of stem cells, growth factors, and diverse soluble mediators. In consequence, some research topics are focused into created enrichment scaffolds. There was only one RTC included in the review related to FGF for REP and it does not demonstrate an impact on clinical success or the increase of root length over BC.

REP denominates procedures designed to replace damaged pulp, root structures, and dentin by physiological mechanisms. The main purpose of REP is to eliminate clinical symptoms and the apical periodontitis resolution. At the same time, the continued root maturation and thickening of the canal walls are secondary objectives [43]. The results of the clinical success of REP were evaluated in the included studies using the following criteria: (1) clinical evidence of periapical healing, (2) radiographic evidence of periapical healing and root development, and (3) response to pulp vitality tests. Regarding the clinical evidence of periapical healing, the absence of sensitivity to percussion, palpation, mobility, and absence of sinus tracts, and inflammation are included; these data should be documented in all clinical reports on REP [44]. The positive response to pulp vitality tests indicates a high level of success, regardless of tissue type generated within the canal [45]. In this review, the results concerning the vitality present inconsistency, which can be explained by the fact that the constituent neoformed tissues are poorly organized. The scientific evidence from histologic studies suggests that the procedures allow for physiological repair instead of regeneration [46].

Preclinical studies on biomimetic materials, bioactive molecules, and stem cells have been promising, however, to date, there is no solid clinical evidence from randomized clinical trials confirming that the additional use of $\mathrm{BC}$ contributes to the outcome of REP. The results from animal studies cannot be extrapolated to clinical conditions. This is because the animal tissues and the pathologies studied are not completely similar to the human ones. Animal studies are highly controlled, which provide high internal validity for these particular conditions studied, but external validity is seriously compromised [47] Likely, a deep understanding of all pulp cell mechanisms and the development of new scaffolds allow the creation of new strategies to increase the success rate of this treatment in the future.

\section{Limitations}

This systematic review encountered some limitations such as small sample sizes, risk of bias, and low certainty of the evidence of the included studies. It is necessary to highlight that distinct treatment protocols were applied even if the implemented scaffolds were simitar; furthermore, the researchers used diverse teeth measurement methods and a diversity of parameters involved between the included studies.

\section{Conclusions}

In the present systematic review, despite the fact that bias was found in the reporting of the results of the included studies, as well as low certainty in the quality assessment, it can conclude that as far as the evidence indicates, the main outcomes under analysis showed no differences between the patients who received novel REP using any type of experimental scaffolds or matrix compared with those that only receive BC.

Supplementary Materials: The following are available online at https: / www.mdpi.com/article/10 .3390 /app11115199/s1, Table S1: title Keywords and algorithms used in the search strategy. 
Author Contributions: R.T.-R. and M.E.M.C.-G. conceived the idea; R.T.-R., M.E.M.C.-G., G.F.-M., A.M.-R., and L.A.-F. collected and analyzed the data; and R.T.-R. and L.A.-F. led the writing. All authors have read and agreed to the published version of the manuscript.

Funding: This research received no specific grant from any funding agency in the public, commercial, or not-for-profit sectors.

Institutional Review Board Statement: Not applicable.

Informed Consent Statement: Not applicable.

Data Availability Statement: Not applicable.

Acknowledgments: R.T.-R., and A.M.-R. thanks to "Cuerpo académico Investigación en Salud"; L.A.-F. thanks to Cátedras CONACyT program. M.E.M.C.-G. Thanks for the scholarship \#755117 from The National Council on Science and Technology (CONACyT).

Conflicts of Interest: The authors declare no conflict of interest.

\section{References}

1. Araújo, P.R.S.; Silva, L.B.; Neto, A.; Almeida de Arruda, J.A.; Álvares, P.R.; Sobral, A.P.V.; Júnior, S.A.; Leão, J.C.; Braz da Silva, R.; Sampaio, G.C. Pulp revascularization: A literature review. Open Dent. J. 2017, 10, 48-56. [CrossRef]

2. Frank, A.L. Therapy for the divergent pulpless tooth by continued apical formation. J. Am. Dent. Assoc. 1966, 72, 87-93. [CrossRef]

3. Andreasen, J.O.; Farik, B.; Munksgaard, E.C. Long-term calcium hydroxide as a root canal dressing may increase risk of root fracture. Dent. Traumatol. 2002, 18, 134-137. [CrossRef]

4. Huang, G.T. Apexification: The beginning of its end. Int. Endod. J. 2009, 42, 855-866. [CrossRef]

5. Nygaard Østby, B. Nyere undersökelser over rotbehandlings-problemet. Odontol. Tidskr. 1963, 71, 467-478.

6. Palma, P.J.; Martins, J.; Diogo, P.; Sequeira, D.; Ramos, J.C.; Diogenes, A.; Santos, J.M. Does apical papilla survive and develop in apical periodontitis presence after regenerative endodontic procedures? Appl. Sci. 2019, 9, 3942. [CrossRef]

7. Diogenes, A.; Hargreaves, K.M. Microbial modulation of stem cells and future directions in regenerative endodontics. J. Endod. 2017, 43 (Suppl. 9), S95-S101. [CrossRef]

8. Kaushik, S.N.; Kim, B.; Walma, A.M.; Choi, S.C.; Wu, H.; Mao, J.J.; Jun, H.W.; Cheon, K. Biomimetic microenvironments for regenerative endodontics. Biomater. Res. 2016, 20, 14. [CrossRef]

9. Alagl, A.; Bedi, S.; Hassan, K.; AlHumaid, J. Use of platelet-rich plasma for regeneration in non-vital immature permanent teeth: Clinical and cone-beam computed tomography evaluation. J. Med. Res. 2017, 45, 583-593. [CrossRef] [PubMed]

10. Higgins, J.P.T.; Green, S. Cochrane Handbook for Systematic Reviews of Interventions; Wiley: Hoboken, NJ, USA, 2011.

11. Sterne, J.A.C.; Savovic, J.; Page, M.J.; Elbers, R.G.; Blencowe, N.S.; Boutron, I.; Cates, C.J.; Cheng, H.Y.; Corbett, M.S.; Eldridge, S.M.; et al. Rob 2: A revised tool for assessing risk of bias in randomised trials. Br. Med. J. 2019, 366, 14898. [CrossRef] [PubMed]

12. Nosrat, A.; Kolahdouzan, A.; Khatibi, A.H.; Verma, P.; Jamshidi, D.; Nevins, A.J.; Torabinejad, M. Clinical, radiographic, and histologic outcome of regenerative endodontic treatment in human teeth using a novel collagen-hydroxyapatite scaffold. J. Endod. 2019, 45, 136-143. [CrossRef]

13. Jadhav, G.; Shah, N.; Logani, A. Revascularization with and without platelet-rich plasma in nonvital, immature, anterior teeth: A pilot clinical study. J. Endod. 2012, 38, 1581-1587. [CrossRef]

14. Bezgin, T.; Yilmaz, A.D.; Celik, B.N.; Kolsuz, M.E.; Sonmez, H. Efficacy of platelet-rich plasma as a scaffold in regenerative endodontic treatment. J. Endod. 2015, 41, 36-44. [CrossRef]

15. Chan, E.K.M.; Desmeules, M.; Cielecki, M.; Dabbagh, B.; Ferraz dos Santos, B. Longitudinal cohort study of regenerative endodontic treatment for immature necrotic permanent teeth. J. Endod. 2017, 43, 395-400. [CrossRef]

16. Li, L.; Pan, Y.; Mei, L.; Li, J. Clinical and radiographic outcomes in immature permanent necrotic evaginated teeth treated with regenerative endodontic procedures. J. Endod. 2017, 43, 246-251. [CrossRef]

17. Kahler, B.; Mistry, S.; Moule, A.; Ringsmuth, A.K.; Case, P.; Thomson, A.; Holcombe, T. Revascularization outcomes: A prospective analysis of 16 consecutive cases. J. Endod. 2014, 40, 333-338. [CrossRef] [PubMed]

18. Sutam, N.; Jantarat, J.; Ongchavalit, L.; Sutimuntanakul, S.; Hargreaves, K.M. A comparison of 3 quantitative radiographic measurement methods for root development measurement in regenerative endodontic procedures. J. Endod. 2018, 44, 1665-1670. [CrossRef]

19. Narang, I.; Mittal, N.; Mishra, N. A comparative evaluation of the blood clot, platelet-rich plasma, and platelet-rich fibrin in regeneration of necrotic immature permanent teeth: A clinical study. Contemp. Clin. Dent. 2015, 6, 63-68. [CrossRef] [PubMed]

20. Nagy, M.M.; Tawfik, H.E.; Hashem, A.A.; Abu-Seida, A.M. Regenerative potential of immature permanent teeth with necrotic pulps after different regenerative protocols. J. Endod. 2014, 40, 192-198. [CrossRef]

21. Shivashankar, V.Y.; Johns, D.A.; Maroli, R.K.; Sekar, M.; Chandrasekaran, R.; Karthikeyan, S.; Renganathan, S.K. Comparison of the effect of prp, prf and induced bleeding in the revascularization of teeth with necrotic pulp and open apex: A triple blind randomized clinical trial. J. Clin. Diagn. Res. 2017, 11, Zc34-zc39. [CrossRef] [PubMed] 
22. Jiang, X.; Liu, H.; Peng, C. Clinical and radiographic assessment of the efficacy of a collagen membrane in regenerative endodontics: A randomized, controlled clinical trial. J. Endod. 2017, 43, 1465-1471. [CrossRef]

23. Santhakumar, M.; Yayathi, S.; Retnakumari, N. A clinicoradiographic comparison of the effects of platelet-rich fibrin gel and platelet-rich fibrin membrane as scaffolds in the apexification treatment of young permanent teeth. J. Indian. Soc. Pedod. Prev. Dent. 2018, 36, 65-70.

24. Ragab, R.A.; Lattif, A.; Dokky, N. Comparative study between revitalization of necrotic immature permanent anterior teeth with and without platelet rich fibrin: A randomized controlled trial. J. Clin. Pediatr. Dent. 2019, 43, 78-85. [CrossRef]

25. Ulusoy, A.T.; Turedi, I.; Cimen, M.; Cehreli, Z.C. Evaluation of blood clot, platelet-rich plasma, platelet-rich fibrin, and platelet pellet as scaffolds in regenerative endodontic treatment: A prospective randomized trial. J. Endod. 2019, 45, 560-566. [CrossRef]

26. Rizk, H.M.; Al-Deen, M.S.S.; Emam, A.A. Regenerative endodontic treatment of bilateral necrotic immature permanent maxillary central incisors with platelet-rich plasma versus blood clot: A split mouth double-blinded randomized controlled trial. Int. J. Clin. Pediatr. Dent. 2019, 12, 332-339. [CrossRef]

27. ElSheshtawy, A.S.; Nazzal, H.; El Shahawy, O.I.; El Baz, A.A.; Ismail, S.M.; Kang, J.; Ezzat, K.M. The effect of platelet-rich plasma as a scaffold in regeneration/revitalization endodontics of immature permanent teeth assessed using 2-dimensional radiographs and cone beam computed tomography: A randomized controlled trial. Int. Endod. J. 2020, 53, 905-921. [CrossRef] [PubMed]

28. Rizk, H.M.; Salah Al-Deen, M.S.M.; Emam, A.A. Comparative evaluation of platelet rich plasma (prp) versus platelet rich fibrin (prf) scaffolds in regenerative endodontic treatment of immature necrotic permanent maxillary central incisors: A double blinded randomized controlled trial. Saudi Dent. J. 2020, 32, 224-231. [CrossRef]

29. Bonte, E.; Beslot, A.; Boukpessi, T.; Lasfargues, J.-J. Mta versus ca(oh)2 in apexification of non-vital immature permanent teeth: A randomized clinical trial comparison. Clin. Oral Investig. 2015, 19, 1381-1388. [CrossRef]

30. Andreasen, F.M.; Andreasen, J.O.; Bayer, T. Prognosis of root-fractured permanent incisors-prediction of healing modalities. Dent. Traumatol. 1989, 5, 11-22. [CrossRef] [PubMed]

31. Ong, T.K.; Lim, G.S.; Singh, M.; Fial, A.V. Quantitative assessment of root development after regenerative endodontic therapy: A systematic review and meta-analysis. J. Endod. 2020, 46, 1856-1866.e2. [CrossRef]

32. Marx, R.E. Platelet-rich plasma: Evidence to support its use. J. Oral Maxillofac. Surg. 2004, 62, 489-496. [CrossRef] [PubMed]

33. Ávila-Curiel, B.X.; Gómez-Aguirre, J.N.; Gijón-Soriano, A.L.; Acevedo-Mascarúa, A.E.; Argueta-Figueroa, L.; Torres-Rosas, R. Intervenciones complementarias para el tratamiento de dolor en pacientes con alteraciones temporomandibulares: Una revisión sistemática. Rev. Int. Acupunt. 2020, 14, 151-159.

34. Franchini, M.; Cruciani, M.; Mengoli, C.; Masiello, F.; Marano, G.; D’Aloja, E.; Dell'Aringa, C.; Pati, I.; Veropalumbo, E.; Pupella, S.; et al. The use of platelet-rich plasma in oral surgery: A systematic review and meta-analysis. Blood Transfus. 2019, 17, 357-367.

35. Nasirzade, J.; Kargarpour, Z.; Hasannia, S.; Strauss, F.J.; Gruber, R. Platelet-rich fibrin elicits an anti-inflammatory response in macrophages in vitro. J. Periodontol. 2020, 91, 244-252. [CrossRef]

36. Kandemir Demirci, G.; Güneri, P.; Çalışkan, M.K. Regenerative endodontic therapy with platelet rich fibrin: Case series. J Clin. Pediatr. Dent. 2020, 44, 15-19. [CrossRef] [PubMed]

37. Bakhtiar, H.; Esmaeili, S.; Fakhr Tabatabayi, S.; Ellini, M.R.; Nekoofar, M.H.; Dummer, P.M. Second-generation platelet concentrate (platelet-rich fibrin) as a scaffold in regenerative endodontics: A case series. J. Endod. 2017, 43, 401-408. [CrossRef]

38. Murray, P.E. Platelet-rich plasma and platelet-rich fibrin can induce apical closure more frequently than blood-clot revascularization for the regeneration of immature permanent teeth: A meta-analysis of clinical efficacy. Front. Bioeng. Biotechnol. 2018, 6, 139. [CrossRef] [PubMed]

39. Riaz, A.; Shah, F.A. Regenerating the pulp-dentine complex using autologous platelet concentrates: A critical appraisal of the current histological evidence. Tissue Eng. Regen. Med. 2021, 18, 37-48. [CrossRef]

40. Babo, P.S.; Pires, R.L.; Reis, R.L.; Gomes, M.E. Membranes for periodontal tissues regeneration. Ciência Tecnol. Mater. 2014, 26, 108-117. [CrossRef]

41. Zeb Khan, S.; Mirza, S.; Karim, S.; Inoue, T.; Bin-Shuwaish, M.S.; Al Deeb, L.; Al Ahdal, K.; Al-Hamdan, R.S.; Maawadh, A.M.; Vohra, F.; et al. Immunohistochemical study of dental pulp cells with $3 \mathrm{~d}$ collagen type i gel in demineralized dentin tubules in vivo. Bosn. J. Basic Med. Sci. 2020, 20, 438-444. [CrossRef]

42. Liu, Q.; Humpe, A.; Kletsas, D.; Warnke, F.; Becker, S.T.; Douglas, T.; Sivananthan, S.; Warnke, P.H. Proliferation assessment of primary human mesenchymal stem cells on collagen membranes for guided bone regeneration. Int. J. Oral. Maxillofac. Implants. 2011, 26, 1004-1010.

43. Staffoli, S.; Plotino, G.; Nunez Torrijos, B.G.; Grande, N.M.; Bossù, M.; Gambarini, G.; Polimeni, A. Regenerative endodontic procedures using contemporary endodontic materials. Materials 2019, 12, 908. [CrossRef] [PubMed]

44. Iwaya, S.I.; Ikawa, M.; Kubota, M. Revascularization of an immature permanent tooth with apical periodontitis and sinus tract. Dent. Traumatol. 2001, 17, 185-187. [CrossRef]

45. Geisler, T.M. Clinical considerations for regenerative endodontic procedures. Dent Clin North Am 2012, 56, 603-626. [CrossRef]

46. Altaii, M.; Richards, L.; Rossi-Fedele, G. Histological assessment of regenerative endodontic treatment in animal studies with different scaffolds: A systematic review. Dent. Traumatol. 2017, 33, 235-244. [CrossRef]

47. Fedele, M.; Gualillo, O.; Vecchione, A. Animal models of human pathology. J. Biomed. Biotechnol. 2011, 2011, 764618. [CrossRef] 